\title{
Radial scaling in inclusive jet production at hadron colliders
}

\author{
Frank E. Taylor* \\ Department of Physics, Laboratory of Nuclear Science, Massachusetts Institute of Technology, \\ Cambridge, Massachusetts 02139, USA
}

(Received 12 December 2017; published 16 March 2018)

\begin{abstract}
Inclusive jet production in $\mathrm{p}-\mathrm{p}$ and $\bar{p}-p$ collisions shows many of the same kinematic systematics as observed in single-particle inclusive production at much lower energies. In an earlier study (1974) a phenomenology, called radial scaling, was developed for the single-particle inclusive cross sections that attempted to capture the essential underlying physics of pointlike parton scattering and the fragmentation of partons into hadrons suppressed by the kinematic boundary. The phenomenology was successful in emphasizing the underlying systematics of the inclusive particle productions. Here we demonstrate that inclusive jet production at the Large Hadron Collider (LHC) in high-energy p-p collisions and at the Tevatron in $\bar{p}-p$ inelastic scattering shows similar behavior. The ATLAS inclusive jet production plotted as a function of this scaling variable is studied for $\sqrt{ } s$ of $2.76,7$ and $13 \mathrm{TeV}$ and is compared to $\bar{p}$ - $p$ inclusive jet production at $1.96 \mathrm{TeV}$ measured at the $\mathrm{CDF}$ and $\mathrm{D} 0$ at the Tevatron and $\mathrm{p}-\mathrm{Pb}$ inclusive jet production at the LHC ATLAS at $\sqrt{ } s_{\mathrm{NN}}=5.02 \mathrm{TeV}$. Inclusive single-particle production at Fermi National Accelerator Laboratory fixed target and Intersecting Storage Rings energies are compared to inclusive $J / \psi$ production at the LHC measured in ATLAS, CMS and LHCb. Striking common features of the data are discussed.
\end{abstract}

DOI: 10.1103/PhysRevD.97.054016

\section{INTRODUCTION}

Single-particle inclusive productions were studied extensively in the early 1970s as a hadronic analogue to deep inelastic electron-nucleon scattering studies conducted at SLAC. The theoretical underpinnings of single-particle inclusive production were developed by Field and Feynman [1], Field et al. [2] and others [3], who described the production of the detected particle to originate from the hard-elastic scattering of a pair of incoming partons which subsequently fragment and hadronize into the inclusively detected particles. The same general quest has been followed in inclusive jet production at hadron colliders (LHC and Tevatron) to test quantum chromodynamics (QCD) and to provide the standard model foundation for searches for a phenomenon beyond the standard model. In the case of inclusive jet production, incoming partons hard scatter, fragment, then hadronize into cones of particles that form jets where the jet itself is analyzed as the inclusively detected particle.

\footnotetext{
* Corresponding author. fet@mit.edu

Published by the American Physical Society under the terms of the Creative Commons Attribution 4.0 International license. Further distribution of this work must maintain attribution to the author(s) and the published article's title, journal citation, and DOI. Funded by SCOAP ${ }^{3}$.
}

Some 40 years ago, in the early time of the operation of the Fermilab synchrotron and at the SPS synchrotron and the Intersecting Storage Ring at CERN, singleparticle inclusive productions, such as $p+p \rightarrow \pi^{0}+X$, $p+p \rightarrow \pi^{ \pm}+X, p+p \rightarrow K^{ \pm}+X$, were studied [4-7]. When the data were analyzed in terms of the transverse momentum $p_{T}$ and the radial scaling variable $x_{R}$, the kinematic form of the Lorentz invariant cross section was greatly simplified. The radial scaling variable is defined by $x_{R}=E / E_{\mathrm{Max}}$, where $\mathrm{E}$ is the detected singleparticle total energy in the center-of-momentum (COM) frame; $E_{\mathrm{Max}}$ is the corresponding maximum energy and is roughly $=\sqrt{ } s / 2$ in the p-p COM frame. The radial scaling variable describes the phase space suppression as the single-particle production approaches the kinematic boundary where $E=E_{\mathrm{Max}}$. Note that this suppression is independent of the angle of the emitted particle in the COM frame and depends only on the radial distance in energy-momentum space to the kinematic boundary.

The earlier analyses of data indicated that the singleparticle inclusive cross section $\mathrm{Ed}^{3} \sigma / \mathrm{dp}^{3}$ had power-law dependences on the transverse momentum $p_{T}$ and on the variable $\left(1-x_{R}\right)$ that roughly factorizes in the form,

$E \frac{d^{3} \sigma}{d p^{3}}=F\left(\sqrt{s}, p_{T}, x_{R}\right) \approx \frac{\alpha}{\left(\Lambda^{2}+p_{T}^{2}\right)^{\frac{n p_{T}}{2}}}\left(1-x_{R}\right)^{n_{x R}}$,

where $\Lambda$ is a transverse mass term, potentially important at low $p_{T}, n_{p T}, n_{x R}$ are the power-law indices and $\alpha$ is the 
parameter that controls the magnitude of the invariant cross section and fixes the dimensions to [momentum $]^{-4}$ (e.g. $\mathrm{GeV}^{-4}$ ). In principle, all the parameters of Eq. (1) can be functions of $\sqrt{ } s$, as well as dependent on the inclusively detected particle. However, in the limited energy range of data analyzed in this earlier work, the s dependence of the inclusive cross section was found to be mostly in the $x_{R}$ variable itself; namely, for fixed $p_{T}$ and $x_{R}$, the inclusive cross sections were roughly constant as $\sqrt{ } s$ was varied. The transverse momentum dependence was found to be approximately independent of the inclusively detected particle, but the $\left(1-x_{R}\right)$ dependence varied for different inclusive particle productions. However, more extensive data taken at the ISR showed that there is an overall s dependence beyond that embodied in the $x_{R}$ variable [8] and this narrowly defined radial scaling was violated. Nevertheless, even with this additional s dependence, the radial scaling formulation was helpful in revealing systematics of the single-particle inclusive cross sections.

The question naturally arises whether or not the radial scaling phenomenology has any utility in uncovering systematics in inclusive jet and charm production in $p-p$ and heavy ion collisions at the LHC. After all, the theoretical underpinning of single-particle inclusive production and jet inclusive production is the same-namely, both are described by hard scattering of incoming partons, followed by fragmentation and hadronization, only in the case of jet production an ensemble of particles carrying the scattered parton momentum is collimated and forms a jet. The following questions are therefore quite natural:

(i) Is the $p_{T}$ dependence of inclusive jet production at the LHC a power law?

(ii) How does the $p_{T}$ dependence of inclusive jet production compare with single-particle inclusive production?

(iii) Is there a power-law dependence in $\left(1-x_{R}\right)$ as was observed in single-particle inclusive processes such as the one given in Eq. (1)?

On general grounds, one may expect that all the parameters of this simple formulation ( $\alpha$ and the power-law indices $n_{p T}$ and $n_{x R}$ ) depend on $\sqrt{ } s$ and that there would be no simplification in the much more complex process of inclusive jet production at $\mathrm{TeV}$ energies. Nevertheless, it is interesting to seek answers to these questions.

\section{JET COLLIDER DATA}

There is now agreement between perturbative quantum chromodynamics (pQCD) calculations to next-to-leading order and inclusive jet production at the LHC to better than $\sim 20 \%$, except at high rapidity and high $p_{T}$, in effect explaining the jet production in terms of scattered partons and subsequent scattered parton hadronizations [9]. It is a success of the underlying theory that the simulations based on $\mathrm{pQCD}$ calculations show such good agreement. Further improvements in the data-theory agreement are expected with the future consideration of higher order effects [10]

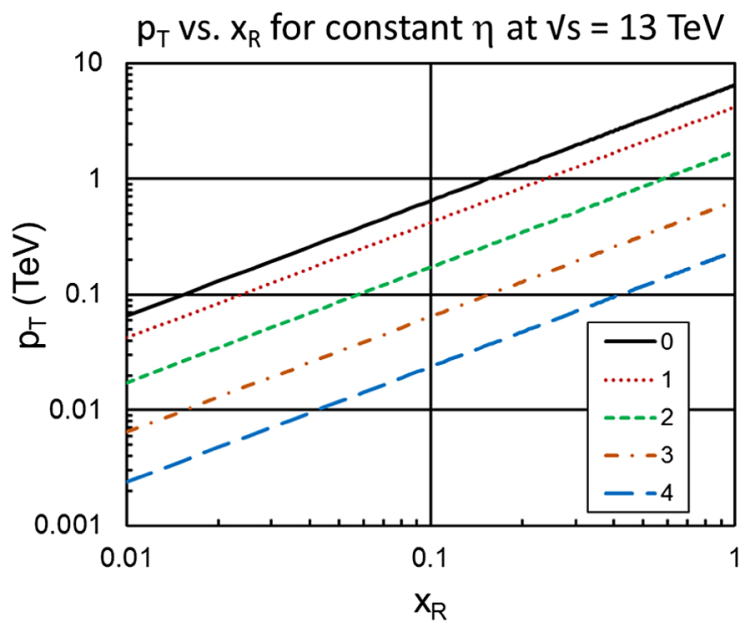

FIG. 1. The lines of constant $\eta$ are plotted on the $p_{T}-x_{R}$ plane for $\sqrt{ } s=13 \mathrm{TeV}$ starting at top $|\eta|=0,1,2,3,4$. The region above and to the left of the $\eta=0$ line (solid black) is kinematically forbidden. Holding $\eta$ (y) constant mixes $p_{T}$ and $x_{R}$ and therefore does not control the radial distance from the kinematic limit $x_{R}=1$.

and better methods of calculating various subprocesses such as by amplitude methods [11].

Inclusive jet production at hadron colliders is conventionally described by $p_{T}$ and the rapidity $y=\frac{1}{2} \ln \left(\frac{E+p_{z}}{E-p_{z}}\right)$, which is roughly equal to the pseudorapidity defined by $\eta=-\ln [\tan (\theta / 2)]$, where $\theta$ is the angle of the emitted jet in the p-p COM frame with respect to the incoming beams. The invariant inclusive jet (single-particle) cross section can by written in terms of the jet transverse momentum, $p_{T}$, and jet rapidity, $\mathrm{y}$, after integrating over the azimuthal angle $\phi$ as

$$
E \frac{d^{3} \sigma}{d p^{3}} \rightarrow \frac{d^{2} \sigma}{p_{T} d p_{T} d y}
$$

In this formulation, the invariant cross section is a function of three variables, the COM energy $\sqrt{ } s$, the transverse momentum $p_{T}{ }^{1}$ and the rapidity $\mathrm{y}$. The jet mass has been integrated into the rapidity variable y through the value of the jet total energy E.

The invariant cross section for inclusive jet or singleparticle production could just as well be written in terms of other groupings of three variables such as $\sqrt{ } s, p_{T}$ and a combination of $\mathrm{y}, p_{T}$ and $\sqrt{ } s$ assembled together to express the radial scaling variable $x_{R}$. In the limit of high energy and small particle or jet mass with respect to $\sqrt{ } s$, the radial scaling variable $x_{R} \approx 2 p_{T} \cosh (\eta) / \sqrt{ } s$, where $\eta$ is the pseudorapidity of the jet in the COM frame. Note that $\cosh (y) \sim \cosh (\eta)=1 / \sin (\theta)$; hence $x_{R} \sim 2 p / \sqrt{ } s \sim$ $E * / E *_{\max }$. Figure 1 shows the relations of the radial

\footnotetext{
${ }^{1}$ We have chosen the $p_{T}$ differential to be $p_{T} \mathrm{dp}_{T}$ rather than $2 p_{T} \mathrm{dp}_{T}=\mathrm{dp}_{T}^{2}$.
} 
scaling variable $x_{R}$ to $p_{T}$ to $\eta$ for $\sqrt{ } s=13 \mathrm{TeV}$. Note that lines of constant $\eta(\eta \sim y)$ mix $p_{T}$ and the scaling variable $x_{R}$. Thus, the kinematic boundary suppression, controlled by $x_{R}$, is convoluted with the $p_{T}$ and $\eta$ (y) dependence.

\section{A. Inclusive jets at the LHC}

It is interesting to analyze inclusive jet production at the LHC in the simplest terms by seeing if there are kinematic generalities like those observed in the single-particle production in p-p collisions. As a typical example, Fig. 2 shows the inclusive jet production at $13 \mathrm{TeV}$ measured by the ATLAS Collaboration at the LHC [12-14].

It is evident that the inclusive jet cross sections agree with the NLOJET $++($ CT14nlo $)[15,16]$ and corrections. However, we note that plotting the cross section for constant $\mathrm{y}$ as a function of $p_{T}$, as in Fig. 2, involves changing the value of $x_{R}$ as was demonstrated in Fig. 1. Therefore, the presentation of the data for constant $y$ obscures a putative power-law behavior in $p_{T}$ and $\left(1-x_{R}\right)$ that we would expect if inclusive jet production in $\mathrm{p}-\mathrm{p}$ scattering has a similar behavior to that of single-particle inclusive cross sections.

Examining Fig. 2, it is evident that the cross section decreases with increasing $p_{T}$ and $\mathrm{y}$, but it is not obvious that there are any power laws in $p_{T}$ and $\left(1-x_{R}\right)$ as were observed in single-particle inclusive production in $\mathrm{p}-\mathrm{p}$ collisions. However, we can roughly test the hypothesis

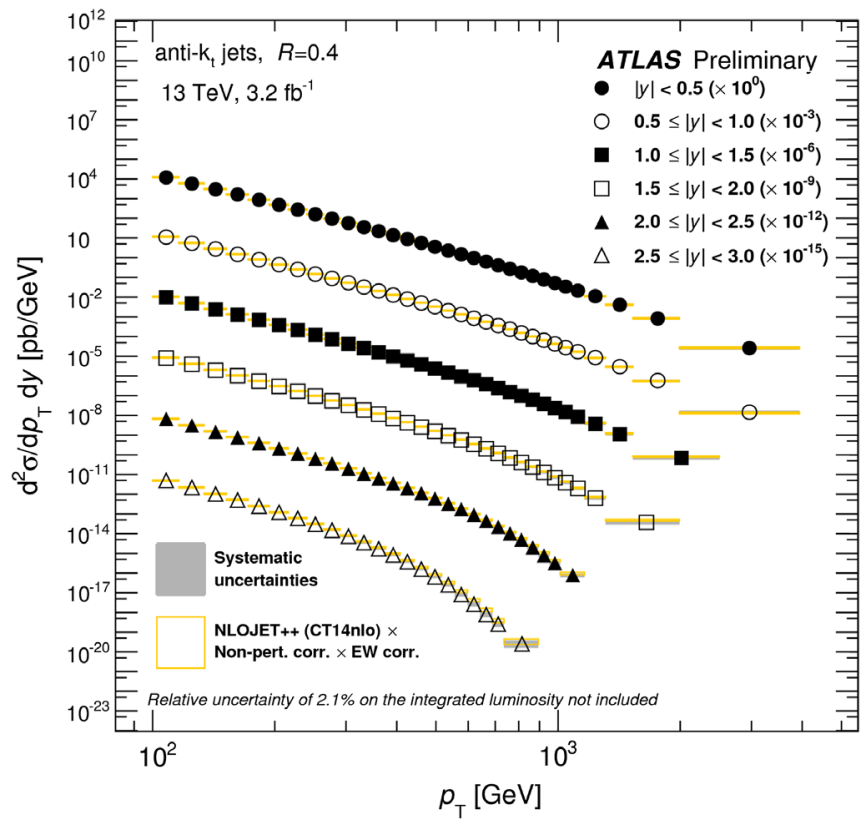

FIG. 2. The ATLAS inclusive jet cross section [12] is plotted as a function of $p_{T}$ for various rapidity regions. The data were taken at $\sqrt{ } s=13 \mathrm{TeV}$ and the jets defined by the anti- $k_{T}$ algorithm. Note that the suppression when close to the kinematic boundary is evident on the rhs of the plot, e.g. for the highest rapidity bin $2.5<|y|<3.0$ the maximum $p_{T}$ is less than $1 \mathrm{TeV} / c$. The data are in good agreement with simulations. that the invariant cross section has the factorized form of Eq. (1) by plotting the resultant invariant cross section $d^{2} \sigma / p_{T} \mathrm{dp}_{T} \mathrm{dy}$ multiplied by a function of $p_{T}$, where we find $\sim p_{T}{ }^{6}$ works reasonably well. The resultant behavior is shown in Fig. 3. Notice that $p_{T}{ }^{6}\left(d^{2} \sigma / p_{T} \mathrm{dp} \mathrm{p}_{T} \mathrm{dy}\right)$ is mostly a function of $x_{R}$ in the sense that the data for different values of $|y|$ fall on top of each other and therefore tend to radially scale for a fixed $\sqrt{ } s$.

For a deeper view of the $p_{T}$ and $x_{R}$ dependencies of the $13 \mathrm{TeV}$ inclusive jet data, we analyze the cross section using the form suggested by single-particle inclusive data,

$$
\frac{d^{2} \sigma}{p_{T} d p_{T} d y}=A\left(p_{T}, s\right)\left(1-x_{R}\right)^{n x_{R}}
$$

by plotting the cross section in slices of constant $p_{T}$ as a function of 1- $x_{R}$. In Eq. (3) we have not assumed a specific form for the $p_{T}$ function, $A\left(p_{T}, s\right)$, except to posit that it is not a function of $x_{R}$. Since the $13 \mathrm{TeV}$ ATLAS inclusive jet data are binned in rapidity (y), thereby requiring the jet mass to be known in order to determine the angle of the jet in the p-p COM, we approximate the radial scaling variable as

$$
\begin{aligned}
x_{R} & =\frac{E}{E_{\max }}=\frac{2 \sqrt{\left(p_{T}^{2} \cosh ^{2}(y)\left(1+\left(m_{J}^{2} / p_{T}^{2}\right) \tanh ^{2}(y)\right)+m_{J}^{2}\right)}}{\sqrt{s}} \\
& \approx \frac{2 p_{T} \cosh (y)}{\sqrt{s}} \sqrt{\left(1+\frac{m_{J}^{2}}{p_{T}^{2}} \tanh ^{2}(y)\right)}
\end{aligned}
$$

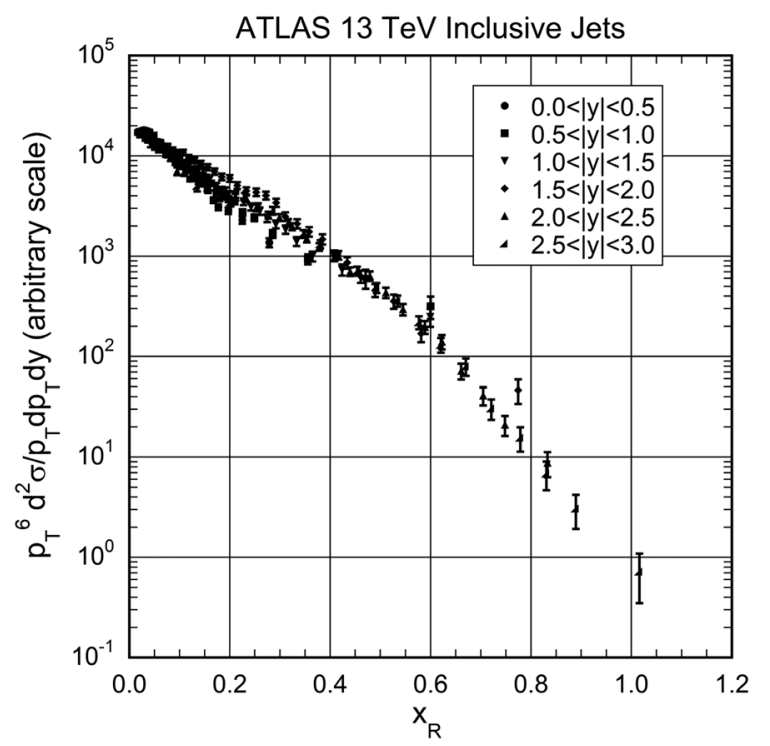

FIG. 3. The $13 \mathrm{TeV}$ ATLAS inclusive jet invariant cross section multiplied by $p_{T}{ }^{6}$ is plotted as a function $x_{R}$ for various $|y|$ values. Note that the data tend to roughly scale as a function of $x_{R}$. The error bars represent the statistical and systematic errors added in quadrature. The point with $x_{R}>1$ is due to the finite bin corrections in $p_{T}$ and y not performed. The data so plotted roughly follow $\left(1-x_{R}\right)^{4.5}$. 
where the jet mass $m_{J}$ has been adsorbed in the variable y but is bounded using the prescription of Ref. [17] by $m_{J} / p_{T}<$ $R / \sqrt{ } 2=0.28$ for the jet cone size $R=0.4$ given by

$$
R=\sqrt{\left(\Delta \phi^{2}+\Delta \eta^{2}\right)},
$$

where $\Delta \phi$ and $\Delta \eta$ are the jet cone widths in $\phi$ and $\eta$, respectively, defined with respect to the colliding beams axis. The finite y bin size was treated by assuming that the published data value for a bin corresponds to the midpoint of the lower and upper limits-a valid assumption for low y, where the rapidity distribution is approximately flat. However, the bin center so calculated is slightly larger by $<1.4 \%$ from a more valid data-weighted value for the highest rapidity bin $(2.5<|y|<3.0)$ resulting in the computed value of $x_{R}$ larger by $<3.8 \%$. This putative finite bin correction was ignored.

The $13 \mathrm{TeV}$ inclusive jet data so analyzed are shown in Fig. 4. We would expect that the $x_{R}$ behavior would be complicated and, even if a power law were operative, the indices $n_{p T}$ and $n_{x R}$ would be functions of $\sqrt{ } s, p_{T}$ and $\mathrm{y}$. Note that $x_{R}=0$, where $A\left(p_{T}, s\right)$ evaluated in the $\left(1-x_{R}\right)$ power-law fits corresponds to the limit when $\sqrt{ } s \rightarrow \infty$ for

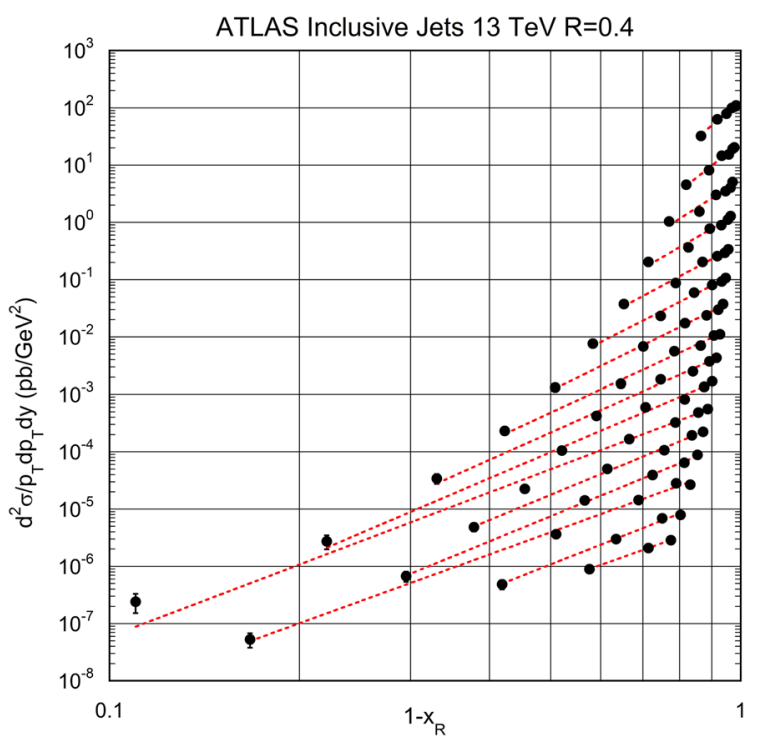

FIG. 4. The $13 \mathrm{TeV}$ ATLAS inclusive jet cross section is plotted as a function of $1-x_{R}$ for various constant values of $p_{T}$. For clarity, only every second value of constant $p_{T}$ of the data set is plotted. Starting at the top of the figure the lines of constant $p_{T}$ are $p_{T}=0.11,0.14,0.18,0.23,0.28,0.33,0.39,0.46,0.53,0.62$. $0.71,0.81,0.92,1.1,1.2,1.4 \mathrm{TeV}$, respectively. The dotted red lines are power-law fits of the form $A\left(p_{T}, s\right)\left(1-x_{R}\right)^{n x R}$ described in the text. Note that the data are consistent with a power law in $\left(1-x_{R}\right)$ as in the case of single-particle inclusive cross sections measured at much lower energies but that the power-law indices $\mathrm{nxR}$ are a function of $p_{T}$. The $\left(1-x_{R}\right)$ power index is larger for lower $p_{T}$. The displayed error bars are statistical and systematic, added in quadrature. The overall error in the luminosity normalization has been neglected. constant $p_{T}$ and thus is beyond the minimum value $(\eta=0)$ of $x_{R \min }=2 p_{T} / \sqrt{ } s$ for finite $\sqrt{ } s$. This small extrapolation assumes that the functional form of Eq. (3) is valid in the small region from $x_{R \min }$ to $x_{R}=0$.

The power-law fits of $\left(1-x_{R}\right)^{n x R}$ were performed by a least-squares linear method on the natural logarithms of the cross section as a function of the $\ln \left(1-x_{R}\right)$ using the statistical and systematic errors added in quadrature. The slopes of these linear fits are the exponents $n_{x R}$ and the constant terms are the logs of $A\left(p_{T}, s\right)$ for the fixed $p_{T}$ values. In general, we would expect that $n_{x R}$ would be a function of $p_{T}$ and $\sqrt{ } s$.

Figure 5 shows the values of $n_{x R}$ plotted as a function of $p_{T}$ where it is evident that $n_{x R} \rightarrow \sim 4$ for high $p_{T}$ but has a higher value for low $p_{T}$. Figure 6 is a plot of $A\left(p_{T}, s\right)$ as a function of $p_{T}$ where it is clear that the data follow a power law as suggested by early radial scaling studies of singleparticle inclusive scattering denoted by Eq. (1) above. The fits, represented by the dotted red lines in the figures below, have the following forms:

$$
\begin{gathered}
n_{x R}\left(p_{T}, s\right)=n_{x R 0}+\frac{D(s)}{p_{T}}, \\
A\left(p_{T}, s\right)=\frac{\alpha(s)}{p_{T}^{n_{p T}}},
\end{gathered}
$$

where $n_{x R 0}$ and $\mathrm{D}$ are the fit parameters for the power of $\left(1-x_{R}\right)$; and $\alpha$ and $n_{p T}$ are the fit parameters for the powerlaw fit to $A\left(p_{T}, s\right)$. Note that at the high $p_{T}$ values of these data the $\Lambda$ term was not necessary.

Encouraged by the simplicity of the $13 \mathrm{TeV}$ inclusive jet cross section when analyzed with the radial scaling variable, we now examine the ATLAS jet data taken at $\sqrt{ } s=2.76$ [18] and $7 \mathrm{TeV}$ [19]. Both analyses at these lower energies used the same anti-kT jet defining algorithm

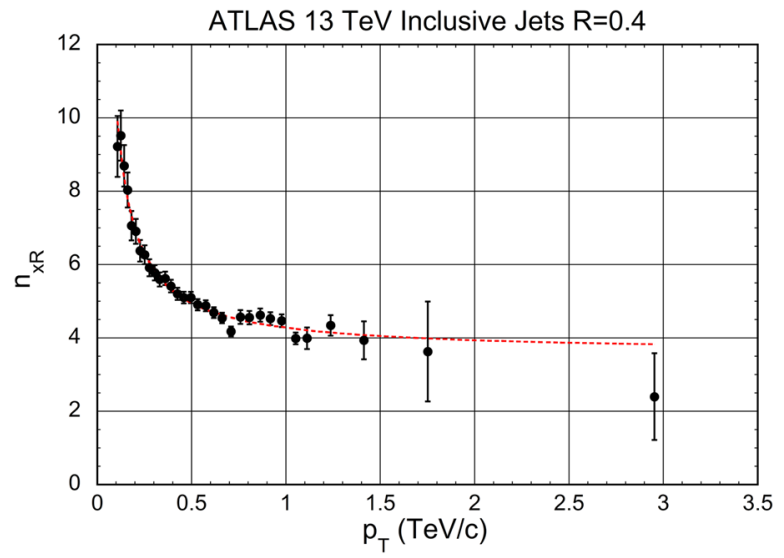

FIG. 5. The exponent of the $\left(1-x_{R}\right)$ power law is shown as a function of $p_{T}$. The red dotted line indicates the fit described in the text that is given by Eq. (6). A $1 / p_{T}$ dependence plus a constant term is evident. 


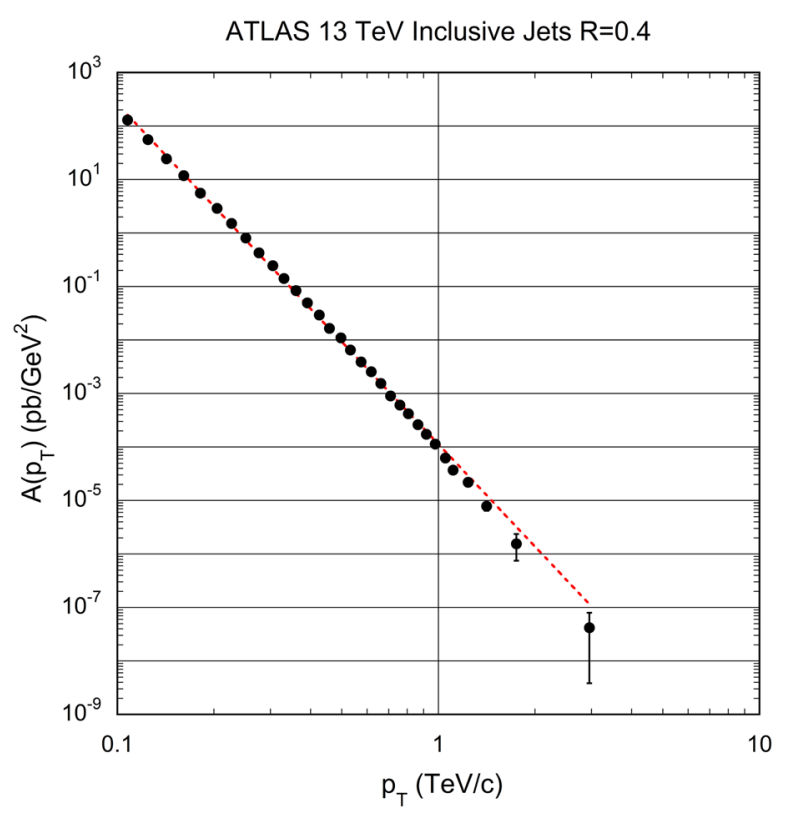

FIG. 6. The fit values $A\left(p_{T}, s\right)$ are plotted as a function of $p_{T}$. Note the power-law behavior over 9 orders of magnitude. The red dotted line indicates the fit $A\left(p_{T}, s\right) \sim 1 / p_{T}{ }^{6.4}$. Note that the $p_{T}$-power index (6.4) is independent of the experimental jet energy scale calibration so long as the energy scale does not depend on the jet energy itself. A small $(\leq \pm 30 \%)$ deviation from the power law is visible and is discussed later.

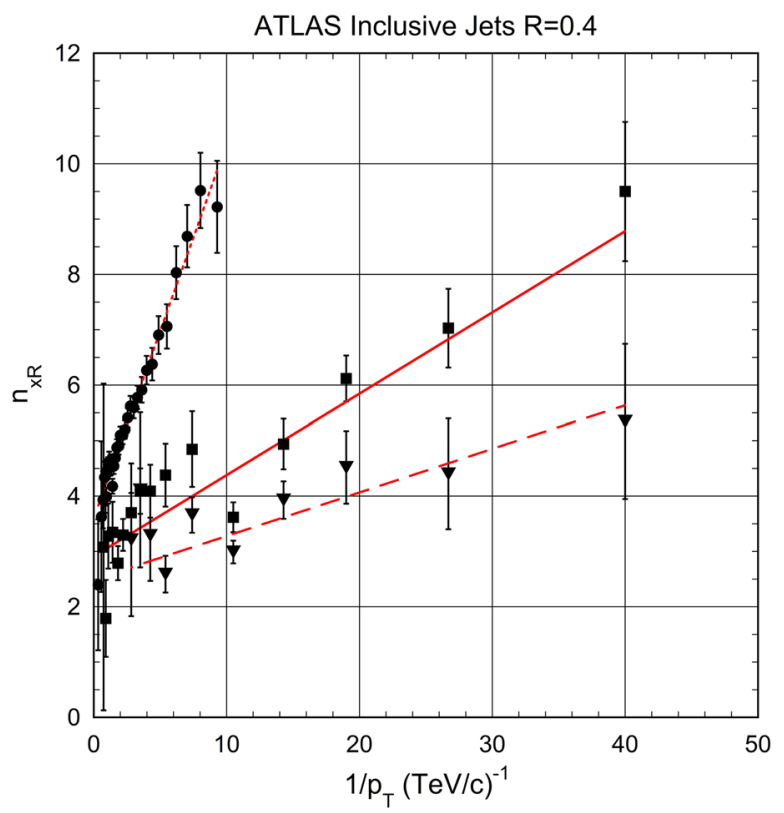

FIG. 7. The exponents of the $\left(1-x_{R}\right)$ power law fits are plotted as a function of $1 / p_{T}$. The ATLAS inclusive jets at $13 \mathrm{TeV}$ are represented by circles, $7 \mathrm{TeV}$ by squares and $2.76 \mathrm{TeV}$ by triangles. The red lines are the straight-line fits in $1 / p_{T}$ of the form given by Eq. (6). Only points with $x_{R}<0.9$ were considered. The power indices are functions of $p_{T}$ and $\sqrt{ } s$ growing with increasing $\mathrm{s}$.

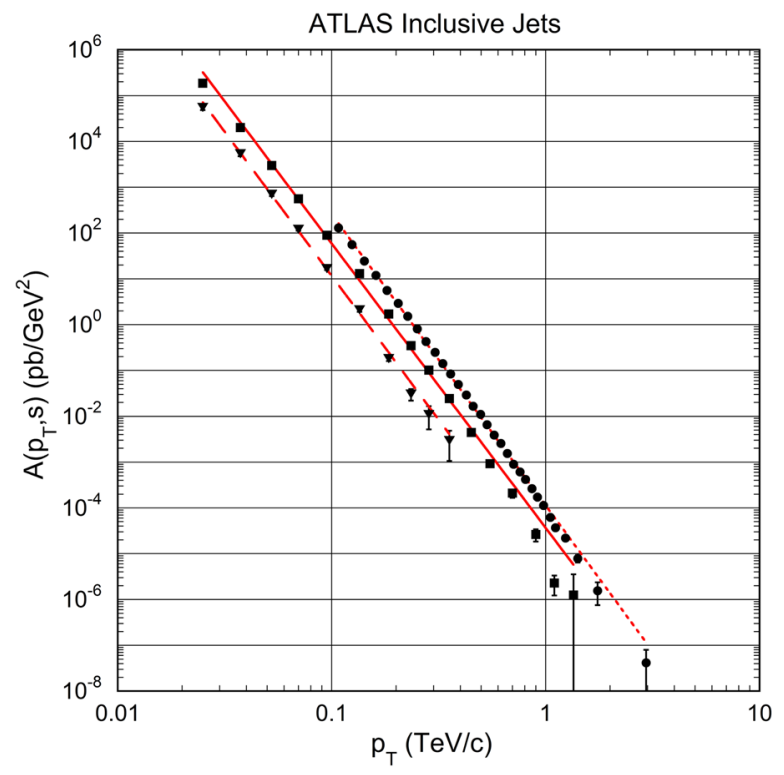

FIG. 8. The $p_{T}$ power law of ATLAS inclusive jet production at the LHC. The jets at $13 \mathrm{TeV}$ are represented by circles, $7 \mathrm{TeV}$ by squares and $2.76 \mathrm{TeV}$ by triangles. The red lines are the power law fits of the form of Eq. (7). We observe that $A\left(p_{T}, s\right)$ functions for the three energies have the same power index, but the overall magnitude of $A\left(p_{T}, s\right)$ grows with increasing s.

as well as the same jet cone definition of $R=0.4$. The resulting power-law plots are shown in Fig. 7 for the $\left(1-x_{R}\right)$ exponent behavior, where we have plotted the exponents as a function of $1 / p_{T}$ to emphasize the linear behavior in that variable, and in Fig. 8 for the $A\left(p_{T}, s\right)$ function. For comparison, the $13 \mathrm{TeV}$ data are plotted on the same scale. Note that the $1 / p_{T}$ term of the $n_{x R}$ dependence grows with increasing $\mathrm{s}$, but the $p_{T}$ power-law exponent, $n_{p T}$, is constant. The overall magnitude of the cross section, governed by the $\alpha$ term, increases with s.

\section{B. CDF and Do inclusive jet $\bar{p}$ p data}

The CDF [20] and D0 [21] inclusive jet data taken at $1.96 \mathrm{TeV}$ collisions $\bar{p}-p$ were analyzed in the same way as the ATLAS inclusive jet data. The results are shown in Figs. 9 and 10. The power indices of $\left(1-x_{R}\right)$ tend to be flatter in rough agreement with the trend seen in Fig. 7, that is, the $1 / p_{T}$ slope of $n_{x R}$ assumes a smaller value for lower COM energies. In Fig. 10 we notice the same $p_{T}$ power-law behavior as seen in p-p inclusive jets. Hence, we conclude that the $p-\bar{p}$ jets have a behavior consistent with trends shown in Figs. 7 and 8 for the LHC p-p jets.

The fit parameters of the data using Eqs. (6) and (7) shown in Figs 7-10 are given in Tables I and II below where we have added a measurement by the CMS Collaboration of inclusive jets at 8 [22] and $13 \mathrm{TeV}$ [23] to the ATLAS measurements discussed above. Notice that the quality of the $n_{x R}$ fit is reasonable $\left(\chi^{2}<1.7 / d . f\right.$. $)$, 


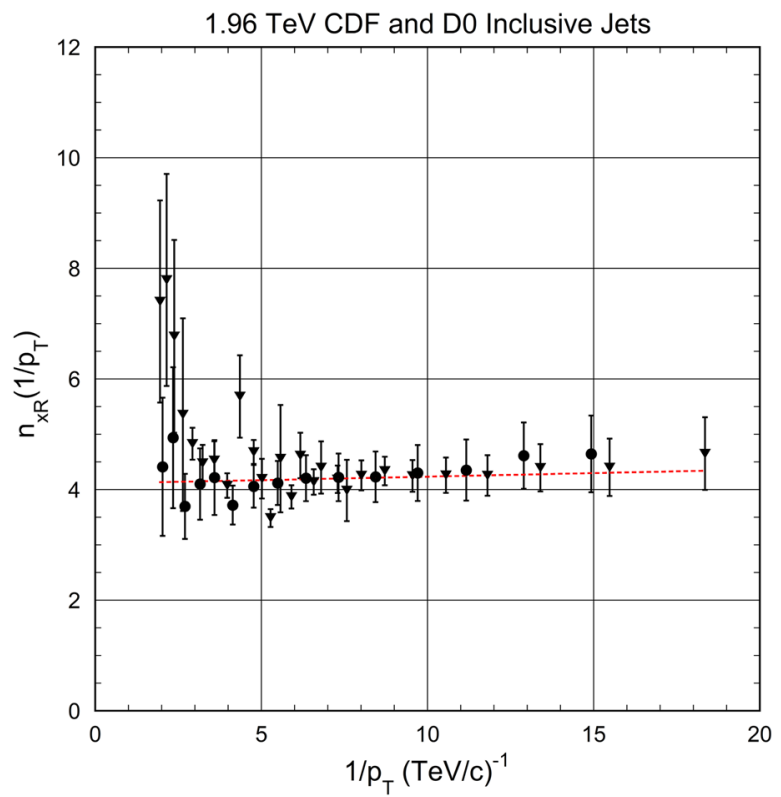

FIG. 9. The exponents of the $\left(1-x_{R}\right)$ power-law fits are plotted as a function of $1 / p_{T}$ for the CDF (circles) and D0 (triangles) inclusive jet production at $1.96 \mathrm{TeV}$ in $\bar{p}-p$ collisions. The dotted red line is the straight-line fits in $1 / p_{T}$ of the form given by Eq. (6) to the CDF and D0 data considered as one data set. The data show considerable scatter, especially at high $p_{T}$ (low $1 / p_{T}$ ). Only data for $x_{R}<0.9$ were included in the fits.

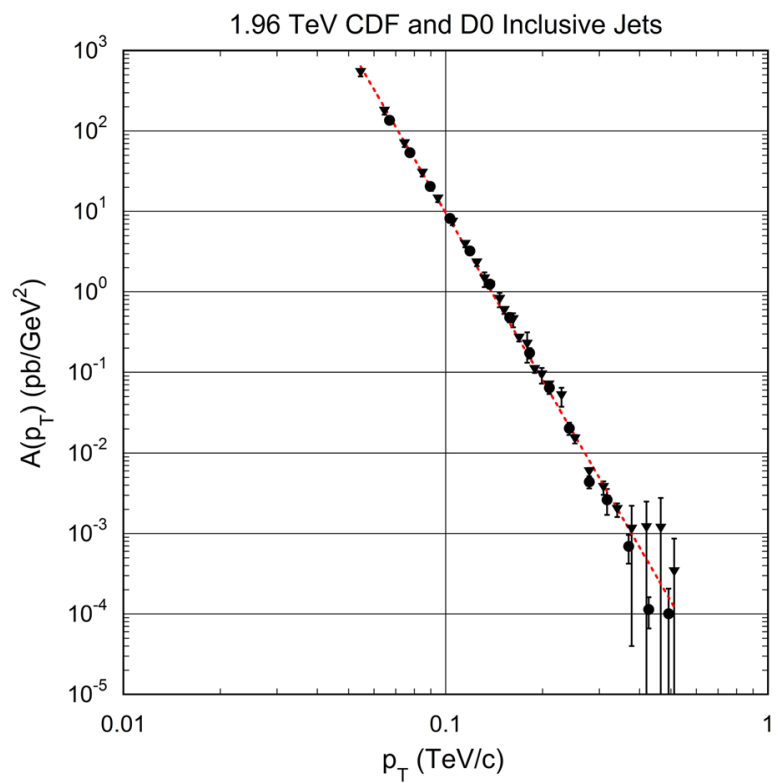

FIG. 10. The $p_{T}$ power law of CDF (circles) and D0 (triangles) for inclusive jet production at the FNAL at $1.96 \mathrm{TeV} \bar{p}$ - $p$ collisions. The systematic and statistical errors have been added in quadrature. The dotted red line is a power-law fit to both data sets taken together. Only data with $x_{R}<0.9$ were considered. The parameters of the fit are in Table II.
TABLE I. The parameters of the fits of the form of Eq. (6) of the power-law indices of the $\left(1-x_{R}\right)^{n x R}$ are tabulated.

\begin{tabular}{lcccr}
\hline \hline \multicolumn{5}{c}{$N_{\mathrm{XR}}$ Fits pp and $P \bar{P}$} \\
\\
\hline$\sqrt{ } s(\mathrm{TeV})$ & $\mathrm{D}\left(\mathrm{TeV}^{-1}\right)$ & $n_{x R 0}$ & $\chi^{2} / d . f$. & d.f. \\
\hline $1.96 \bar{p}-p$ CDF & $0.06 \pm 0.04$ & $3.7 \pm 0.3$ & 0.2 & 13 \\
$1.96 \bar{p}-p$ D0 & $0.00 \pm 0.02$ & $4.2 \pm 0.2$ & 2.0 & 25 \\
2.76 p-p ATLAS & $0.08 \pm 0.03$ & $2.5 \pm 0.3$ & 1.2 & 8 \\
5.02 p-Pb p-side ATLAS & $0.07 \pm 0.02$ & $3.2 \pm 0.2$ & 0.8 & 13 \\
7 p-p ATLAS & $0.15 \pm 0.02$ & $2.9 \pm 0.2$ & 1.7 & 14 \\
8 p-p CMS & $0.22 \pm 0.01$ & $2.96 \pm 0.03$ & 1.2 & 33 \\
13 p-p ATLAS & $0.68 \pm 0.03$ & $3.61 \pm 0.07$ & 0.8 & 30 \\
13 p-p CMS & $0.34 \pm 0.09$ & $3.5 \pm 0.2$ & 0.3 & 27 \\
\hline \hline
\end{tabular}

whereas that of the power-law fit to $A\left(p_{T}, s\right)$ has a large $\chi^{2} / d$.f. This is discussed later.

Examining Tables I and II we conclude that most of the variation of the parameters of these fits to inclusive jet production is in the overall normalization term controlled by the parameter $\alpha$, which increases with increasing $\sqrt{ } s$, and the $p_{T}$ dependence of the power of $\left(1-x_{R}\right)$ given by the term, D, which also increases with increasing $\sqrt{ } s$. The parameters $\mathrm{np}_{T}$ and $\mathrm{nx}_{R} 0$ do not show such large systematic $\sqrt{ } s$ dependences.

The parameters shown in Tables I and II are plotted in Fig. 11 below. It is interesting to note that the $\mathrm{D}$ and $\alpha$ terms grow linearly with $\mathrm{s}$, whereas the $n_{x R 0}$ and $n_{p T}$ terms are roughly constant. The units of $\alpha$ in Table II are $\left[\mathrm{pb} / \mathrm{GeV}^{2} \mathrm{TeV}^{n p T}\right]$, which for $n_{p T} \sim 6$ become [energy ${ }^{2}$, the same units as the Mandelstam variable, s. Hence it is not surprising that $\alpha$ grows linearly with increasing $\mathrm{s}$ in order to preserve the dimensions of the invariant cross section $d^{2} \sigma / p_{T} \mathrm{dp} \mathrm{p}_{T} \mathrm{dy}$ to be $\left[\mathrm{pb} / \mathrm{GeV}^{2}\right]$.

\section{Inclusive jet production in $\mathbf{p}-\mathrm{Pb}$ collisions}

For another view of inclusive jet production at the LHC we analyze the jet data taken in $\mathrm{p}-\mathrm{Pb}$ collisions at a nucleon-nucleon COM energy of $\sqrt{ } s_{\mathrm{NN}}=5.02 \mathrm{TeV}$ [24]. Here we examine the two sides of the collision separately-namely the side where the incoming proton

TABLE II. The parameters of the power-law fits to $A\left(p_{T}, s\right)$ according to Eq. (7) are tabulated. The $\chi^{2} / d$.f . values are not very likely and are discussed later.

\begin{tabular}{lcccr}
\hline \hline \multicolumn{5}{c}{$P_{T}$ fits pp and $P \bar{P}$} \\
\hline$\sqrt{ } s(\mathrm{TeV})$ & $\alpha\left(\mathrm{pb} / \mathrm{GeV}^{2}\right) \mathrm{TeV}^{n p T}$ & \multicolumn{1}{c}{$n_{p T}$} & $\chi^{2} / d . f$. & d.f. \\
\hline $1.96 \bar{p}-p$ CDF & $(0.9 \pm 0.2) \times 10^{-6}$ & $7.03 \pm 0.08$ & 4 & 13 \\
$1.96 \bar{p}-p$ D0 & $(1.3 \pm 0.1) \times 10^{-6}$ & $6.90 \pm 0.05$ & 1.2 & 25 \\
2.76 p-p ATLAS & $(6.0 \pm 1.0) \times 10^{-6}$ & $6.29 \pm 0.06$ & 3.4 & 8 \\
7 p-p ATLAS & $(3.7 \pm 0.2) \times 10^{-5}$ & $6.21 \pm 0.03$ & 32 & 14 \\
8 p-p CMS & $(2.98 \pm 0.04) \times 10^{-5}$ & $6.73 \pm 0.01$ & 28 & 33 \\
13 p-p ATLAS & $(1.13 \pm 0.02) \times 10^{-4}$ & $6.36 \pm 0.01$ & 8 & 30 \\
13 p-p CMS & $(1.06 \pm 0.04) \times 10^{-4}$ & $6.40 \pm 0.03$ & 2 & 27 \\
\hline \hline
\end{tabular}


(a)
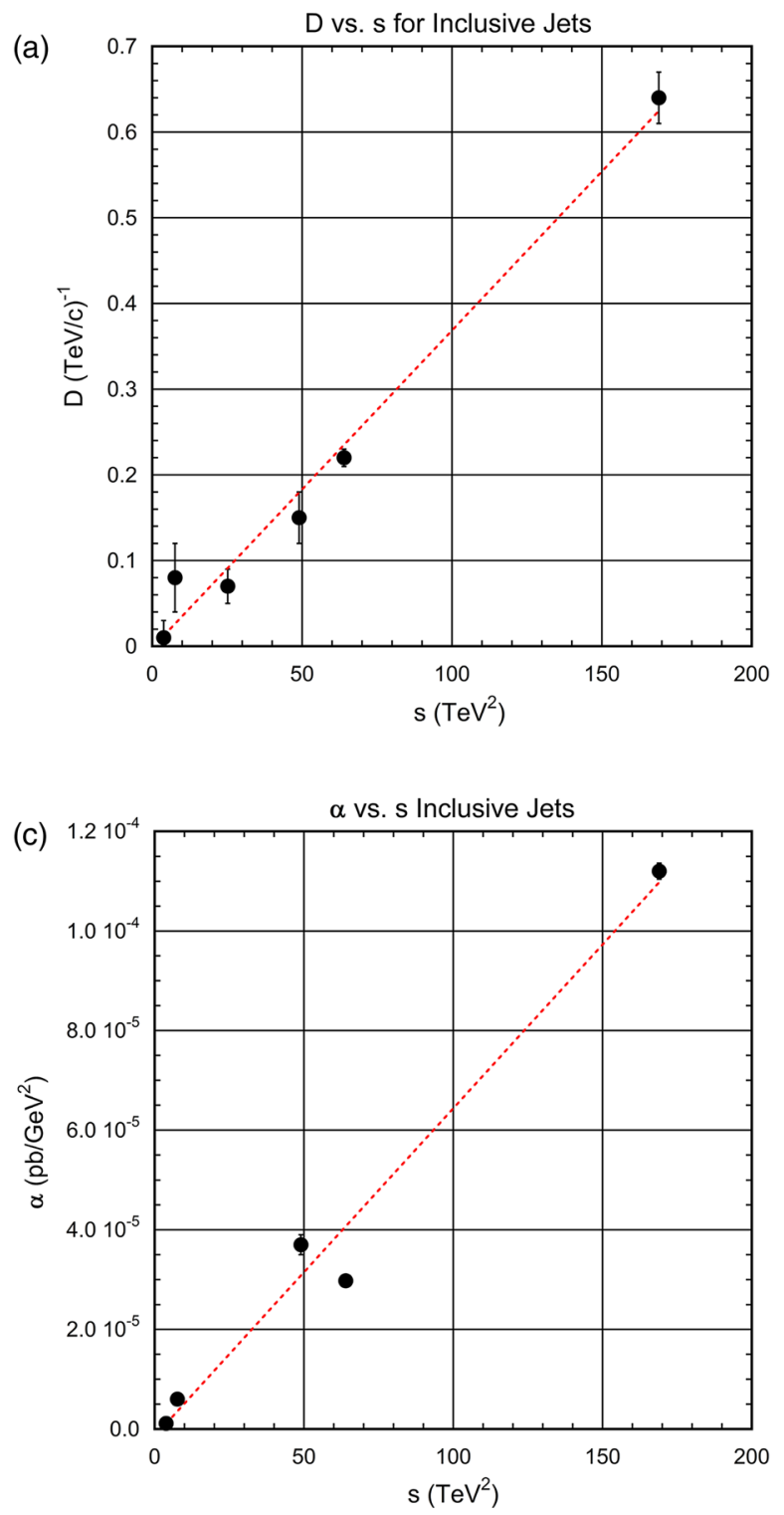
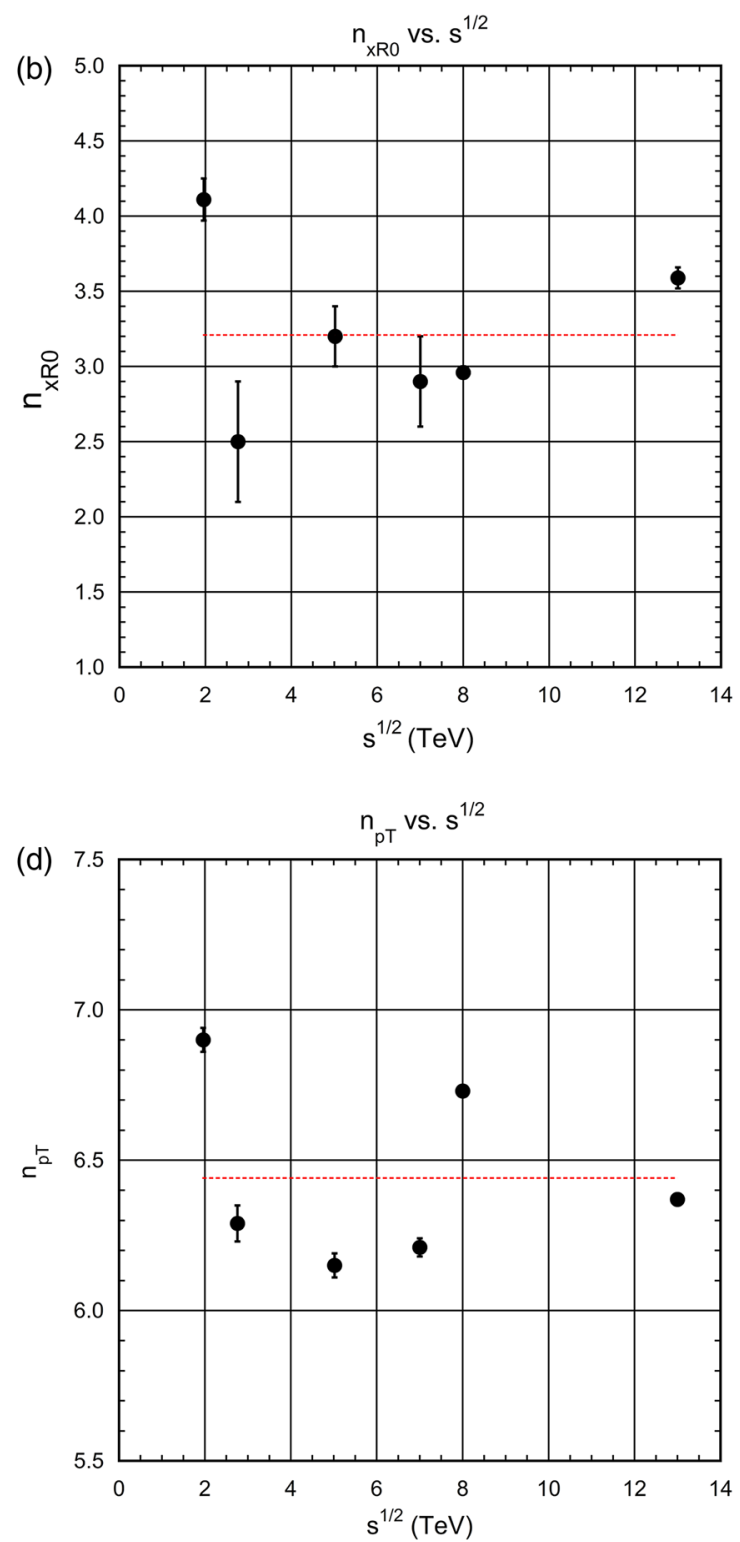

FIG. 11. [(a) and (b)] The 1- $x_{R}$ power index parameters are plotted vs $\sqrt{ } s$. (a): $\mathrm{D}(\mathrm{s})$ appears to grow linearly with $\mathrm{s}$ as indicated by the red-dotted line; whereas (b) $n_{x R 0}$ is roughly constant (red dotted line indicates the average). (c) The cross section magnitude $\alpha(s)$ is plotted, which scales linearly in s. Note that the dimension (TeVnpT) shown in Table II is implicit in the plot by the convention that $p_{T}$ is always expressed in units of $\mathrm{TeV} / c$ to yield a cross section in $\left(\mathrm{pb} / \mathrm{GeV}^{2}\right)$. (d) $n_{p T}$ is constant where the red dotted line indicates the average. The CDF and D0 parameters at $1.96 \mathrm{TeV}$ have been combined by weighted average by their respective errors. The $13 \mathrm{TeV}$ ATLAS and CMS values are combined similarly. The $\mathrm{p}$ - $\mathrm{Pb}$ values at $\sqrt{ } s=5.02 \mathrm{TeV}$ [24] are shown except in the $\alpha(s)$ plot, where no value can be determined since the cross section was self-normalized.

fragments $(y>0)$ and the side where the $\mathrm{Pb}$ nucleus fragments $(y<0)$. Rather than arbitrarily assigning the central rapidity bin $-0.3<y<0.3$ to either the proton forward or the $\mathrm{Pb}$-forward data, this central bin was included in both sides of the data. If there were an underlying hard partonparton scattering that initiates the formation of the detected jet, we would naively expect the same power law in the transverse momentum $p_{T}$ as observed in jet production in p-p collisions. On the other hand, the fragmentation part of the cross section expressed by the $x_{R}$ dependence may be different since the jet formation on the $\mathrm{Pb}$ fragmentation side would have to contend with many nucleus fragments, whereas the jet formation on the proton side would be similar to p-p scattering. A difference would be an expression of the well-established jet quenching [25] observed in heavy ion collisions.

The comparison of inclusive jets in $\mathrm{p}-\mathrm{Pb}$ scattering of the power of $\left(1-x_{R}\right)$ for the two fragmentation cases is shown in Fig. 12. The corresponding $p_{T}$ dependences are shown in Fig. 13. The fit parameters are listed in Tables III and IV. 
ATLAS Inclusive Jets 5.02 TeV p-Pb Scattering

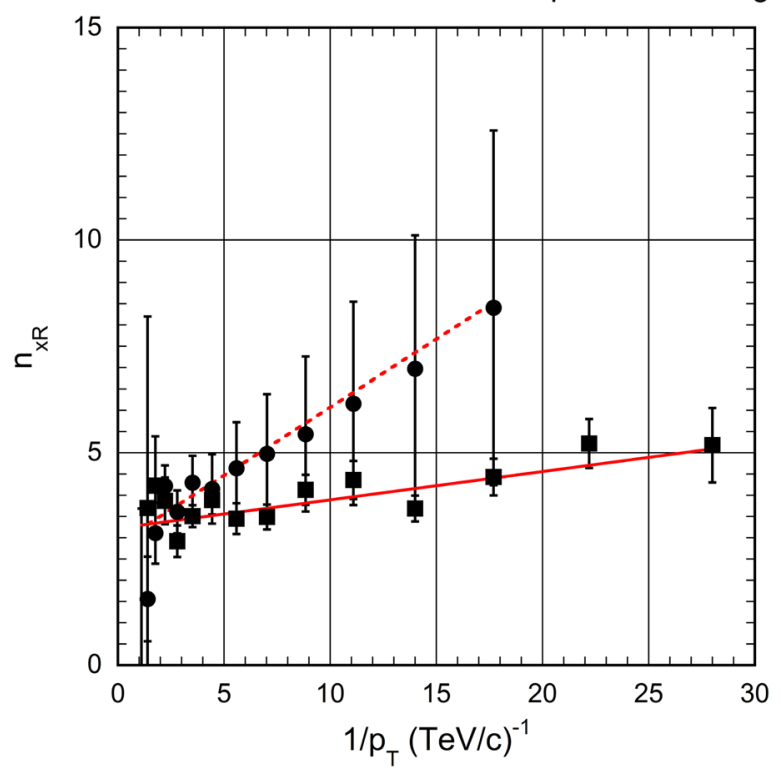

FIG. 12. The exponents of the $\left(1-x_{R}\right)$ power-law fits for the two sides of the ATLAS p-Pb inclusive jet data taken at $\sqrt{ } s_{\mathrm{NN}}=$ $5.02 \mathrm{TeV}$. The closed circles correspond to the $\mathrm{Pb}$-forward data and the closed squares to the p-forward side. The red lines are the fits of the form given by Eq. (6). Note that the $\mathrm{Pb}$ fragmentation side has a steeper $1 / p_{T}$ dependence than the proton fragmentation side. The error bars represent the statistical and systematic errors added in quadrature. The systematic errors dominate.

From Tables III and IV we conclude that the $p_{T}$ dependences are approximately the same in the two fragmentation cases (the values of $n_{p T}$ are within $5 \%$ of each other), whereas the $\mathrm{D}$ terms of the $\left(1-x_{R}\right)$ exponent parametrizations by Eq. (6) depend strongly on the type of fragmentation side. In fact, such a $\mathrm{D}$ value for the $\mathrm{Pb}$ fragmentation side would correspond to inclusive jets at $\approx 10 \mathrm{TeV}$ in p-p collisions, implying that the quenching of jets observed in heavy ion collisions is also operative in p-p collisions, but at higher energies. This interpretation suggests an equivalency between the formation and quenching of jets at lower energies in A-A collisions with jet production at higher energies in $p-p$ collisions and could be systematically studied by performing this analysis for jets produced in A-A collisions as a function of centrality.

\section{Comparison with inclusive jet simulations}

It is not the object of this paper to appraise the quality of the $\mathrm{pQCD}$ simulations of inclusive jet production, but it is of interest to check that the simulations show the same power-law behaviors. Of the data examined in this work, from the CDF inclusive jets at $1.96 \mathrm{TeV}$ published in 2009 to ATLAS inclusive jets at $13 \mathrm{TeV}$ published in 2016 there is good agreement with simulations. The CDF analysis used the midpoint jet clustering algorithm with a cone size $R=0.7$ and proton and antiproton parton distribution functions (PDFs) from [26] in conjunction with PYTHIA 6.2
ATLAS $5.02 \mathrm{TeV}$ p-Pb Inclusive Jets

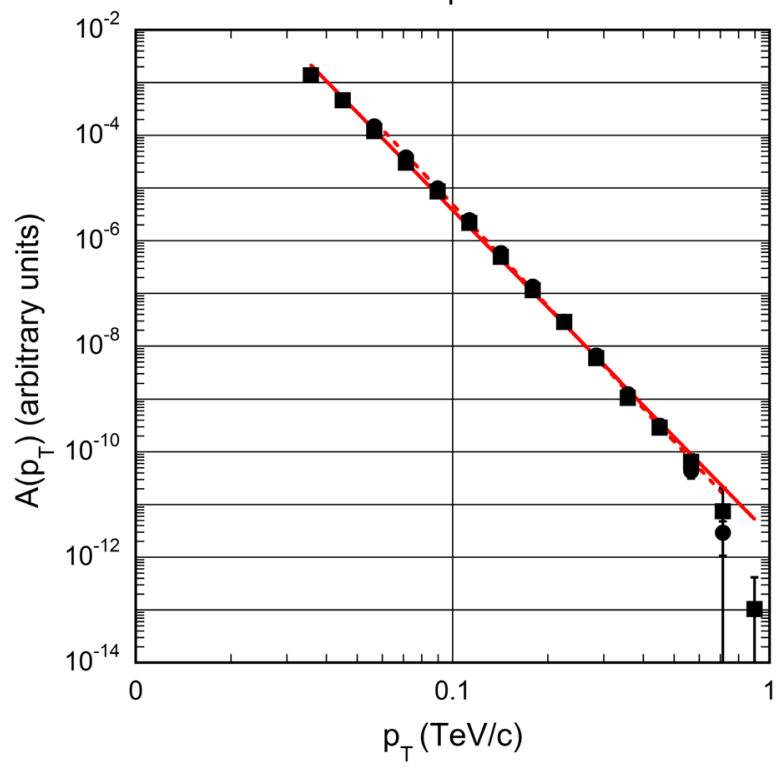

FIG. 13. The $p_{T}$ power law and fits for the two sides of the ATLAS p-Pb inclusive jet data taken at $\sqrt{ } s_{\mathrm{NN}}=5.02 \mathrm{TeV}$. The closed circles correspond to the Pb-forward data and the closed squares to the $\mathrm{p}$-forward side. The red lines are the fits of the form given by Eq. (7). Note that the $p_{T}$ dependence is consistent within errors. As in Fig. 12, the error bars represent the statistical and systematic errors added in quadrature. The systematic errors dominate. $A\left(p_{T}, s\right)$ units are arbitrary since the cross section is self-normalized.

[27]. The ATLAS Collaboration used an anti- $k_{T}$ clustering algorithm with $R=0.4$ and a more refined PDF set in PYTHIA $8.186[28,29]$.

As a demonstration of the agreement, the Monte Carlo (MC) simulation SHERPA [30] has been compared with the $7 \mathrm{TeV}$ ATLAS data, where the MC data were analyzed in the same way as the ATLAS $7 \mathrm{TeV}$ inclusive jet data using the radial scaling formulation. The comparison of the ratios (MC/data) of the respective fit parameters is given in Table V.

All parameters of the data-SHERPA comparison are consistent with each other, with the exception of D, which is smaller by about $30 \%$ in the SHERPA simulation $(3 \sigma)$. However, we note that this comparison of data vs MC

TABLE III. The parameters of the fits of the form of Eq. (6) of the power-law indices of the $\left(1-x_{R}\right)^{n x R}$ of constant $p_{T}$ of Eq. (6) are tabulated. Notice that the $p_{T}$ dependence in the $\mathrm{D}$ term for $\mathrm{Pb}$-forward data is four times larger (roughly 3 standard deviations) than that of the p-forward case, whereas the $n_{x R 0}$ value is the same within errors.

\begin{tabular}{lcccr}
\hline \hline \multicolumn{5}{c}{$N_{\mathrm{XR}}$ FITS $P-P_{B}$} \\
\hline$\sqrt{ } s(\mathrm{TeV})$ & $\mathrm{D}\left(\mathrm{TeV}^{-1}\right)$ & $n_{x R 0}$ & $\chi^{2} / d . f$. & d.f. \\
\hline $5.02 \mathrm{p}$-side & $0.07 \pm 0.02$ & $3.2 \pm 0.2$ & 0.8 & 13 \\
$5.02 \mathrm{~Pb}$-side & $0.3 \pm 0.1$ & $2.9 \pm 0.5$ & 0.6 & 10 \\
\hline \hline
\end{tabular}


TABLE IV. The parameters of the power-law fits to $A\left(p_{T}, s\right)$ according to Eq. (7) are tabulated. The power indices $\mathrm{np}_{T}$ are the same within $5 \%$ for the p-forward and $\mathrm{Pb}$-forward cases and are consistent with the index for $\mathrm{p}$-p scattering given in Table II.

\begin{tabular}{lccc}
\hline \hline \multicolumn{4}{c}{$P_{T}$ FITS $P-P_{B}$} \\
\hline$\sqrt{ } s(\mathrm{TeV})$ & $n_{p T}$ & $\chi^{2} / d . f$. & d.f. \\
\hline $5.02 \mathrm{p}$-side & $6.15 \pm 0.04$ & 25 & 13 \\
$5.02 \mathrm{~Pb}$-side & $6.43 \pm 0.07$ & 6 & 10 \\
\hline \hline
\end{tabular}

TABLE V. The ratio of the fit parameters of the SHERPA simulation of the $7 \mathrm{TeV}$ ATLAS inclusive jet data is given.

\begin{tabular}{lc}
\hline \multicolumn{2}{c}{ SHERPA-data comparison } \\
\hline Parameter & Ratio (SHERPA/data) \\
\hline$\alpha$ & $1.2 \pm 0.3$ \\
$n_{p T}$ & $0.98 \pm 0.02$ \\
$\mathrm{D}$ & $0.7 \pm 0.1$ \\
$n_{x R} 0$ & $1.06 \pm 0.09$ \\
\hline \hline
\end{tabular}

using the $p_{T}$ and $x_{R}$ variables is quite sensitive to $\mathrm{y}$ dependence and may be a useful test of data/MC in the future.

\section{SINGLE-PARTICLE INCLUSIVE DATA}

Since we find that the inclusive jet production at the LHC in $\mathrm{p}-\mathrm{p}, \mathrm{p}-\mathrm{Pb}$ collisions and in $\bar{p} p$ collisions at the Fermi National Accelerator Laboratory (FNAL) collider has power law dependences in both $p_{T}$ and $\left(1-x_{R}\right)$, it is interesting to analyze single hadron and prompt photon production.

\section{a. Prompt photon production}

In prompt photon production, the photon is believed to come directly from the primordial hard parton scattering such as q $g \rightarrow q \gamma$ and higher order processes, such as the fragmentation process q $g \rightarrow q \mathrm{~g} \gamma$. Unlike jet production, prompt photon production has no final state interaction other than the radiative fragmentation process above. Hence, the $E_{T}$ dependence as well as the $\left(1-x_{R}\right)$ dependence are important measures of the production mechanism without the influence of the final state processes.

A number of authors (for example see [31]) have extensively analyzed direct photon production in $\mathrm{p}-\mathrm{p}$ collisions as a means to determine the nucleon gluon distribution, but not with our variables $\left(p_{T}, x_{R}\right)$. For this study, we consider the prompt photon data determined by CMS at $7 \mathrm{TeV}$ [32] and that of ATLAS at 8 [33] and $13 \mathrm{TeV}$ [34]. The photon data are analyzed in the same manner as the inclusive jet data-namely we compute the invariant cross sections $d^{2} \sigma / E_{T} \mathrm{dE}_{T} d \eta$ and plot the results as a function of $\left(1-x_{R}\right)$ for fixed $E_{T}$ in order to determine

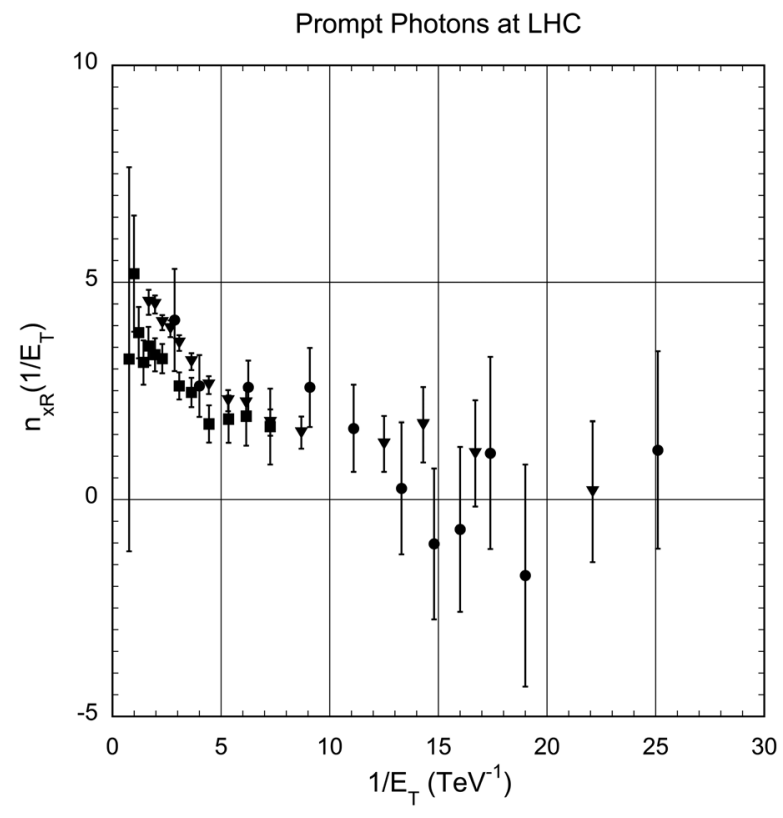

FIG. 14. Shown are the $\left(1-x_{R}\right)$ exponents as a function of $1 / E_{T}$ of the analyses of the $7 \mathrm{TeV}$ CMS prompt photon data (closed circles) and the 8 (triangles) and $13 \mathrm{TeV}$ (squares) data sets of ATLAS. The highest point in both the $7 \mathrm{TeV}$ CMS data and $8 \mathrm{TeV}$ ATLAS data is weighted averages of the points where the errors are larger than $100 \%$ plotted at the weighted $1 / E_{T}$ value. The error bars are the quadrature sums of statistical and systematic contributions.

$A\left(E_{T}, s\right)$ and the $\left(1-x_{R}\right)$ power-law indices. The outcomes of the analysis for all three data sets are shown in Figs. 14 and 15.

As in the analysis of the inclusive jet data, we have determined the power indices $n_{x R}$ of Fig. 14 by fits of the function of Eq. (6), where $E_{T}$ replaced $p_{T}$ and the function $A\left(E_{T}, s\right)$ in Fig. 15 with Eq. (7). The results are given in Tables VI and VII. It is interesting to note in Table VI that the parameter $\mathrm{D}$ for prompt photons is negative and appears to grow more negative with increasing $\sqrt{ } s$. This is in contrast with the behavior for inclusive jets at the LHC where $\mathrm{D}$ is positive and increases with increasing $\sqrt{ } s$. The parameter $n_{x R} 0$ has an average value $\left\langle n_{x R} 0\right\rangle=4.2 \pm 0.4$ which does not show a systematic energy dependence, although the dispersion of the data is large.

In Table VII, where values of $\alpha$ and $n_{E T}$ are given, we see that the overall direct photon cross section grows with increasing $\sqrt{ } s$ as indicated by the fitted values of the $\alpha$ parameter. This is the same general behavior observed in our analysis of inclusive jet production cross sections.

It is notable that the power-law index $n_{E T}$ is less than the corresponding value for inclusive jets. Averaging over the three measurements in Table VII ( 7 to $13 \mathrm{TeV}$ ) we find $\left\langle n_{\mathrm{ET}}\right\rangle=5.6 \pm 0.2$ unweighted average, whereas the average of the corresponding parameter for inclusive jets in the energy range 7 to $13 \mathrm{TeV}$ is $\left\langle n_{p T}\right\rangle=6.4 \pm 0.2$. This suggests that the prompt photon leaves the scene of the primordial collision unencumbered; whereas jets must 
Prompt Photons at LHC

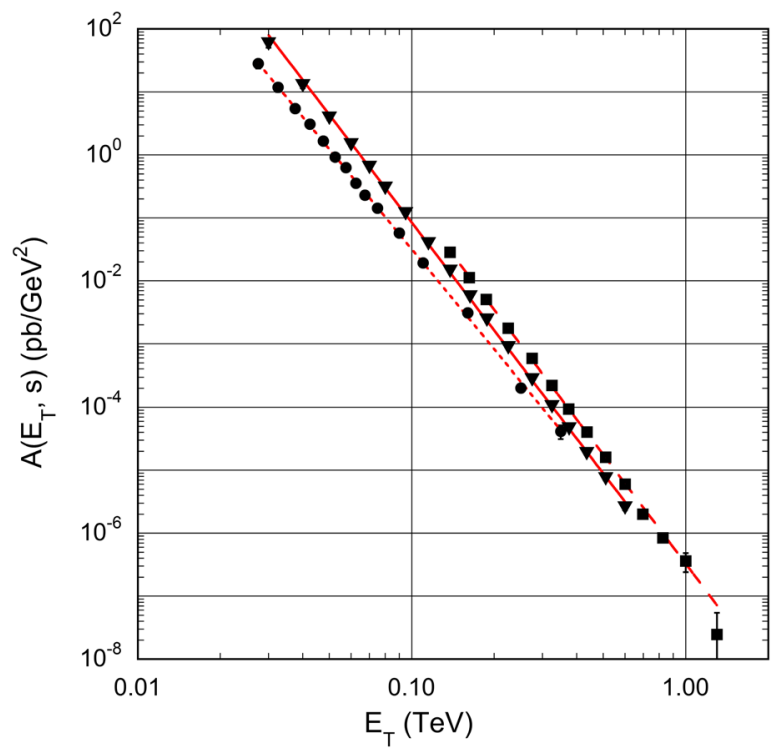

FIG. 15. The function $A\left(E_{T}, s\right)$ is plotted for the $7 \mathrm{TeV}$ CMS prompt photon data (circles) and the 8 and $13 \mathrm{TeV}$ data of ATLAS (triangles and squares, respectively). The power-law fits given in Table VII are shown in the red dotted line for $7 \mathrm{TeV}$ CMS, and solid and dashed lines for 8 and $13 \mathrm{TeV}$ ATLAS measurements, respectively.

tear themselves free of the QCD color fields. If we assume that $E_{T}$ dependence of $A\left(E_{T}, s\right)$ for prompt photons is a measure of the primordial hard parton scattering, then the fragmentation and hadronization operative in the production of jets in $\mathrm{p}-\mathrm{p}$ and $p-\bar{p}$ collisions contribute to the jet and hadron $p_{T}$ dependence by roughly one more term $\sim 1 / p_{T}$ (a $3 \sigma$ difference with these data).

TABLE VI. The prompt photon invariant cross section parameters of the fits of the form of Eq. (6) of the power-law indices of the $\left(1-x_{R}\right)^{n \times R}$ for constant $E_{T}$ are tabulated.

\begin{tabular}{lcccc}
\hline \hline \multicolumn{5}{c}{$N_{\text {XR }}$ FITS DIRECT PHOTON } \\
\hline$\sqrt{ } s(\mathrm{TeV})$ & $\mathrm{D}\left(\mathrm{TeV}^{-1}\right)$ & $n_{x R 0}$ & $\chi^{2} /$ d.f. & d.f. \\
\hline $7 \mathrm{TeV}$ CMS & $-0.20 \pm 0.07$ & $3.8 \pm 0.7$ & 0.4 & 13 \\
$8 \mathrm{TeV}$ ATLAS & $-0.35 \pm 0.03$ & $4.7 \pm 0.1$ & 2.5 & 16 \\
$13 \mathrm{TeV}$ ATLAS & $-0.43 \pm 0.09$ & $4.1 \pm 0.3$ & 0.4 & 12 \\
\hline \hline
\end{tabular}

TABLE VII. The prompt photon invariant cross section parameters of the power-law fits to $A\left(E_{T}, s\right)$ according to Eq. (7) are tabulated.

\begin{tabular}{lcccc}
\hline \hline \multicolumn{5}{c}{$P_{T}$ FITS DIRECT PHOTON } \\
\hline$\sqrt{ } s(\mathrm{TeV})$ & $\alpha\left(\mathrm{pb} / \mathrm{GeV}^{2}\right) \mathrm{TeV}^{n E T}$ & $n_{E T}$ & $\chi^{2} / d . f$. & d.f. \\
\hline $7 \mathrm{TeV}$ CMS & $(1.7 \pm 0.2) \times 10^{-7}$ & $5.28 \pm 0.05$ & 0.7 & 13 \\
$8 \mathrm{TeV}$ ATLAS & $(1.72 \pm 0.05) \times 10^{-7}$ & $5.69 \pm 0.01$ & 2.8 & 16 \\
$13 \mathrm{TeV}$ ATLAS & $(3.3 \pm 0.1) \times 10^{-7}$ & $5.76 \pm 0.03$ & 1.4 & 12 \\
\hline \hline
\end{tabular}

\section{b. Hadron production}

A host of other inclusive production data were analyzed in the same way. Since the data extend to lower transverse momenta, we expect the parton intrinsic transverse momentum $\left(k_{T}\right)$ to be an influence as well as transverse mass effects for heavy particle production. Moreover, for the case of charm production at the LHC we might expect a similar term that could arise from a production mechanism from the decay of a heavier "parent" particle. Hence, we fit the $p_{T}$ dependence with the form given in Eq. (8) below,

$$
A(p T)=\frac{\alpha}{\left(\Lambda^{2}+p_{T}^{2}\right)^{\frac{n_{p T}}{2}}},
$$

where the values of $\Lambda, \alpha$ and $n_{p T}$ are determined by a minimum $\chi^{2}$ fit [35]. A typical result is shown in Fig. 16 for $\pi^{+}$using the compilation of Refs. [5].

The results of the $p_{T}$ power-law fits are listed in Table VIII. We note that all the processes considered, including the five direct photon measurements, have an average $p_{T}$ power-law dependence $A\left(p_{T}, s\right) \sim 1 / p_{T}{ }^{n p T}$ with an index $\mathrm{np}_{T} \approx 6.1 \pm 0.6$. The inclusive production of light hadrons up to $K^{+}$has a $\Lambda$ value consistent with the parton intrinsic $k_{T} \sim 0.6 \mathrm{GeV}$ [36], whereas the $J / \psi$ and $\psi(2 S)$ production are consistent with a larger $\Lambda$ value $(\Lambda \geq 3.6 \mathrm{GeV})$ which must provide an important clue about their production mechanism [37].

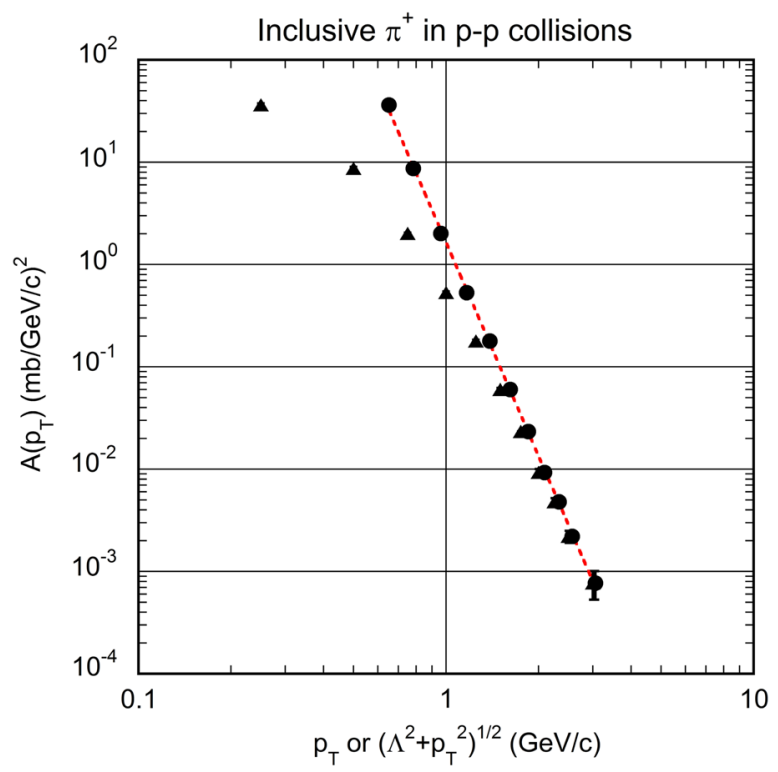

FIG. 16. The inclusive $\pi^{+}$data [5] are plotted with respect to the measured $p_{T}$ (black triangles) and with respect to $\left(\Lambda^{2}+p_{T}^{2}\right)^{1 / 2}$ closed black circles. The red dotted line is the power-law fit corresponding to $\mathrm{np}_{T}=6.94 \pm 0.04$ as given in Table VIII. A minimum $\chi^{2}$ fit of $\Lambda$ from Eq. (8) yields minimum at $\Lambda=0.602 \pm 0.012 \mathrm{GeV}$ consistent with intrinsic parton $k_{T}$ of the nucleon [36]. 
TABLE VIII. Tabulated are the values of the power-law fits to various processes through Eq. (8). All processes are for inclusive production in p-p collisions except for Ag-Ag collisions of BRAHAMS RHIC data [38]. For those entries of the table where $\sqrt{ } s=0.063 \mathrm{TeV}$ the tabulated $\sqrt{ } s$ value is the maximum of the data set, which also includes lower $\sqrt{ } s$ values down to $10 \mathrm{GeV}$ in some entries [5]. The values of $\Lambda$ and associated errors are determined by the curvature $\chi^{2}$ function about its minimum. The 7 TeV CMS prompt $J / \psi$ data $[39,40]$ are consistent with $\Lambda=0$, unlike the other measurements, but with a large error and for this reason $\Lambda$ and $\sigma(\Lambda)$ for this entry are left blank. Entries 1 through 5 for direct $\gamma$ have a minimum $E_{T} \gg k_{T} \sim 0.6 \mathrm{GeV}$ and thus no sensitivity to the $k_{T}(\Lambda$ ) value. The last column indicates the average value of $\Lambda$ for each category of inclusive processes.

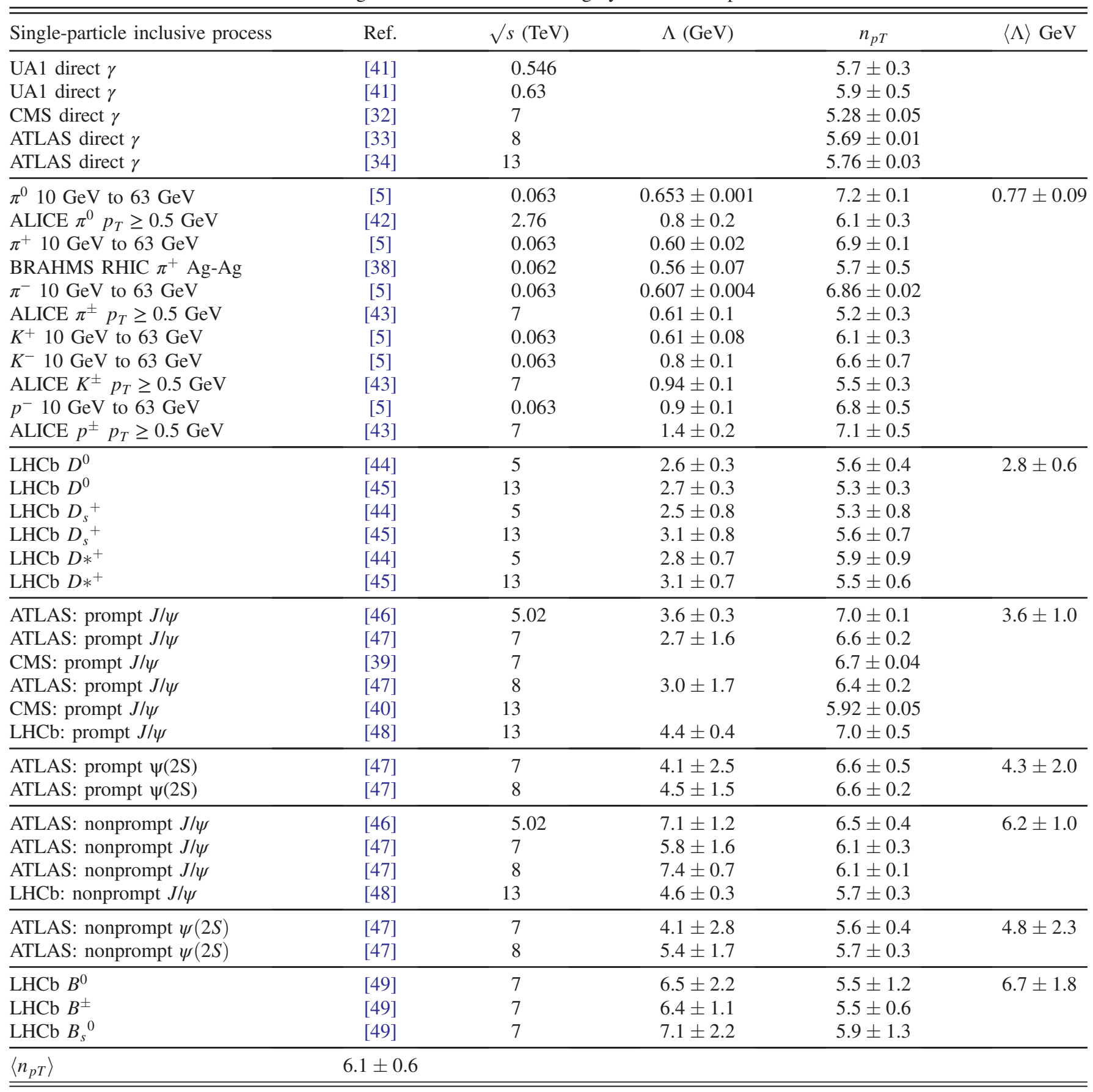

The power-law indices average $=6.1 \pm 0.6$. The values of $\Lambda$ for the single light $\left(\pi^{ \pm, 0}, K^{+}\right)$particle inclusive production are consistent with the intrinsic $k_{T}$. Note that
$\langle\Lambda\rangle$ tends to grow with increasing mass of the inclusively detected particle, and tends to be larger for $J / \psi$ nonprompt production in comparison to $J / \psi$ prompt production. 


\section{LINE COUNTING, HIGHER TWISTS, AND DIQUARKS}

Using the radial scaling formulation discussed above and examining Tables II, IV, and VIII, it is remarkable that the $p_{T}$ factorized part of the invariant cross sections is a power law with the behavior $A\left(p_{T}, s\right) \approx \alpha(s) / p_{T}{ }^{6}$ with essentially all the s dependence confined in the term $\alpha(s)$. This is true for inclusive jet production in high-energy $\mathrm{p}-\mathrm{p}, \quad \bar{p}-p$ collisions and inclusive single-particle production in $p-p$ and inclusive $\pi^{+}$production in Ag-Ag collisions. (Direct photon production favors $\sim 1 / p_{T}^{5.6 \pm 0.2}$.) Further, the exponent $n_{x R}$ of $\left(1-x_{R}\right)$ for LHC inclusive jet production is found to be a linear function of $1 / p_{T}$ with the slope parameter $\mathrm{D}$ increasing with increasing $\sqrt{ } s$. Inclusive jet production in $\mathrm{p}-\mathrm{Pb}$ collisions shows the same behavior but has a significantly different $\mathrm{D}$ value depending on the fragmentation side (proton forward or the $\mathrm{Pb}$ forward).

It is well known that the dimensions of the invariant cross section for partons are dependent on the number of active fields that hard scatter to produce the detected jet or the particle in nucleon-nucleon scattering. By this argument the matrix element for the hard-scattering $M \sim$ (Mass $^{4-n A}$, where $\mathrm{nA}$ is the number of active fields that scatter [50-54] and $\hat{s}$ is the total parton-parton COM energy squared. Since the invariant cross section has the form given by Eq. (9) we would expect the $p_{T}$ dependence of the invariant cross section by this argument to be given by Eq. (10).

$$
\begin{gathered}
\frac{d^{2} \sigma}{p_{T} d p_{T} d y} \propto \frac{|M|^{2}}{\hat{s}^{2}} \\
\frac{d^{2} \sigma}{p_{T} d p_{T} d y} \propto \frac{1}{p_{T}^{2 n_{A}-4}}
\end{gathered}
$$

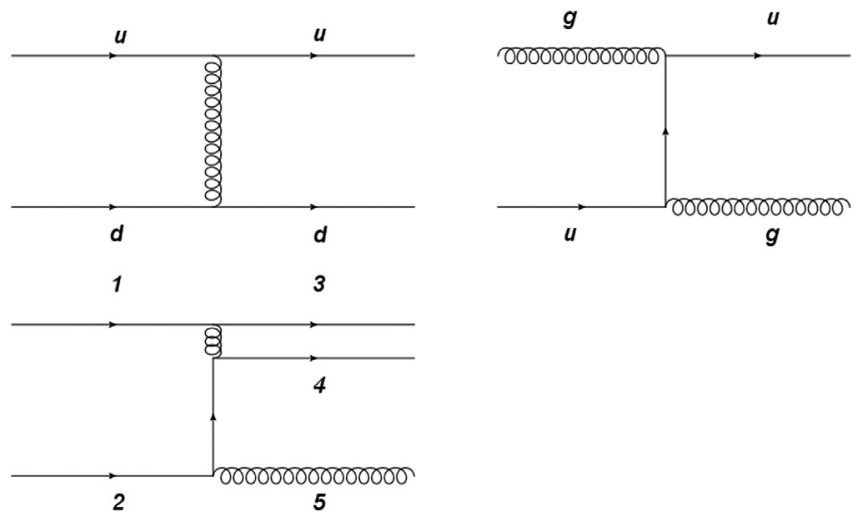

FIG. 17. Shown are Feynman diagrams for u-d quark elastic scattering, g-u elastic scattering and quark-quark scattering with two gluons, one radiated (line 5) to the final state. Note that lines 3 and 4 denote a diquark, where the number of active lines $n_{A}=5[52]$.
Referring to Fig. 17 below, we note, for example, that u-d elastic scattering, or g-u elastic scattering, involves four active fields and therefore would have a $p_{T}$ dependence given by Eq. (11a). Whereas, for example the diagram at the bottom-left part of the figure involves quark-quark scattering with two radiated gluons, one in the final state and one that forms a diquark. This diagram involves five fundamental fields and consequently would have a $p_{T}$ dependence given by Eq. (11b).

$$
\begin{gathered}
\frac{d^{2} \sigma}{p_{T} d p_{T} d y} \propto \frac{1}{p_{T}^{4}} \\
\frac{d^{2} \sigma}{p_{T} d p_{T} d y} \propto \frac{1}{p_{T}^{6}} .
\end{gathered}
$$

It is noteworthy that all the processes tabulated above seem to favor the $p_{T}$ dependence given in Eq. (11b) over a wide range of energies, rather than the lowest order scattering which has a $p_{T}$ dependence given by Eq. (11a). Other diagrams, such as ones with a radiated gluon from a scattered quark, would correspond to a $2 \rightarrow 3$ scattering resulting in a higher $p_{T}$ power. The $p_{T}$ power-law index $\sim 6$ is a surprise since one would expect an index of $\sim 4$ for parton-parton $(2 \rightarrow 2)$ hard elastic scattering at lowest order.

A number of authors have observed [55-60]that the effective $p_{T}$ power is larger than the expected dimensional limit of $2 \rightarrow 2$ scattering but some researchers find that the $p_{T}$ power seems to depend on the process. Some of these analyses explore the limit to scaling as a function of $x_{T} \rightarrow 0$, which we have shown does not respect the kinematic boundary and therefore mixes the kinematic boundary suppression with the underlying $p_{T}$ dependence. An appraisal of one of these studies [51] is given in the Appendix. On the contrary, we find that the average $p_{T}$ power is $\left\langle n_{p T}\right\rangle=6.2 \pm 0.6$ for all 48 inclusive single photon/hadron/jet data sets considered in $\mathrm{p}-\mathrm{p}, \bar{p}-p, \mathrm{p}-\mathrm{Pb}$ collisions (for inclusive jets in $\mathrm{p}-\mathrm{p}$, $\bar{p}-p$ and $\mathrm{p}-\mathrm{Pb}\left\langle n_{p T}\right\rangle=6.5 \pm 0.3$ (Tables II and IV) and single-particle inclusive cross sections $\left\langle n_{p T}\right\rangle=$ $6.1 \pm 0.6)$ (Table VIII). The invariant cross section dimensional limit $n_{p T}=4$ is therefore disfavored by $3.8 \sigma$. Even direct $\gamma$ production disfavors $n_{p T}=4$ by $8 \sigma$ $\left(\left\langle n_{E T}\right\rangle=5.6 \pm 0.2\right.$ vs 4$)$ (Table VII). This behavior is consistent with a dominant $2 \rightarrow 3$ hard scattering that is saturated at a relatively low $\sqrt{ } s$, thereby becoming independent of process and COM energy (see Feynman et al. [2]).

The dominant $2 \rightarrow 3$ scattering is consistent with an intrinsic diquark inside the nucleon, although a $2 \rightarrow 2$ scattering with a radiated gluon from one of the final quark legs would also have a cross section of the same $p_{T}$ dimension. Evidence of diquark correlations in the proton has been discussed for some time [61]. Recently, data from 


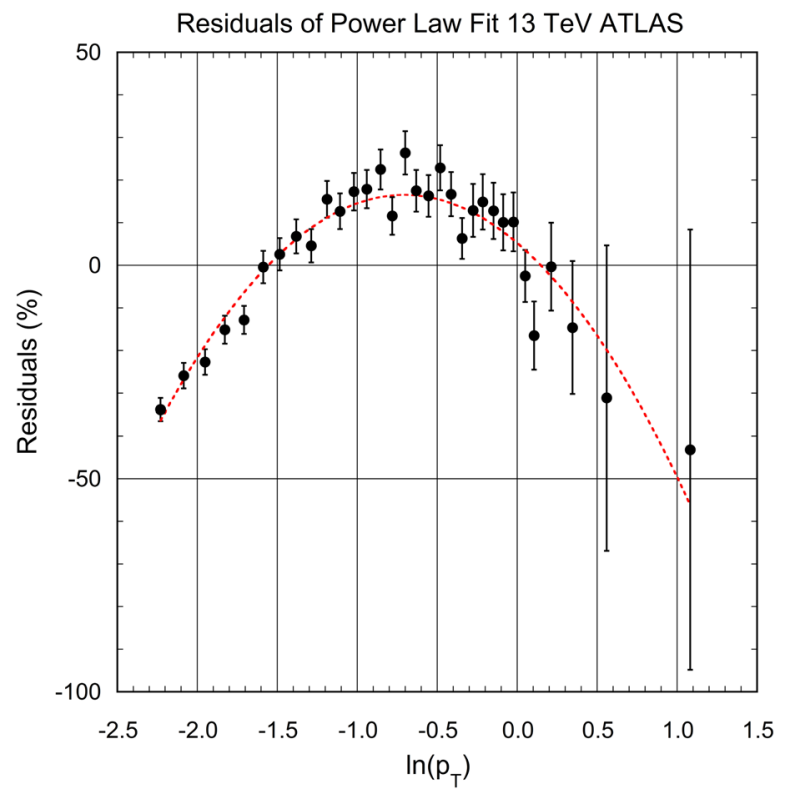

FIG. 18. The residuals of the power-law fit to $13 \mathrm{TeV}$ ATLAS inclusive jet data. A simple power law $\sim 1 / p_{T}{ }^{6.45 \pm 0.037}$ fits the data $\left(\chi^{2} /\right.$ d.f. $=8.2$ for 30 d.f.). The residuals are confined to be within $\sim \pm 30 \%$ over 9 orders of magnitude in $p_{T}$. The dotted red line is a quadratic fit in $\ln \left(p_{T}\right)$ to the residuals.

JLab [62] supported the notion of diquarks affecting the proton elastic form factors. Lattice QCD calculation also indicates that there is a strong association of the u-d quarks in the proton that forms a singlet (diquark) state $[63,64]$.

\section{V. $A\left(p_{T}, s\right)$ FOR JETS AS A QUADRATIC IN $\ln \left(p_{T}\right)$}

We have noted in Tables II and IV that the $p_{T}$ powerlaw fits for inclusive jets had rather unlikely $\chi^{2}$ values. A close examination of the fit-data relation reveals a systematic deviation from a pure power law-namely, there is a small curvature making the $p_{T}$ dependence less steep at low $p_{T}$ than at high $p_{T}$. The effect is illustrated in Fig. 18 where we plot the residuals of the power-law fit of the $13 \mathrm{TeV}$ ATLAS inclusive jet data as a function of $\ln \left(p_{T}\right)$. In order to make the discrepancy clear, the baseline power-law fit was determined by treating all errors the same. The error bars in the figure represent the statistical and systematic errors added in quadrature as in Fig. 6.

We notice that the residuals of the single power-law fit plotted in Fig. 18 can be quite well fitted to a quadratic in $\ln \left(p_{T}\right)$. This suggests that the $p_{T}$ dependence of the invariant cross section for inclusive jets at $13 \mathrm{TeV}$ is a function of the type

$\ln \left(A\left(p_{T}, s\right)\right)=\beta(s) \ln \left(p_{T}\right)^{2}-n_{p_{T}} \ln \left(p_{T}\right)+\rho(s)$.

An equivalent form of Eq. (12) makes evident the underlying $p_{T}$ power law with a moderating term controlled by the parameter $\beta$ and is given by

$$
A\left(p_{T}, s\right)=\exp \left(\beta(s)\left(\ln \left(p_{T}\right)\right)^{2}\right) \frac{\alpha(s)}{p_{T}^{n_{p_{T}}}},
$$

where $\alpha(s)=\exp (\rho(s))$.

Fitting $A\left(p_{T}, s\right)$ of ATLAS and CMS inclusive jets and the inclusive jets of CDF and D0 1.96 TeV to Eq. (12), we find the parameter values given in Table IX. The residuals of the ATLAS $13 \mathrm{TeV}$ inclusive jets of this quadratic $\ln \left(p_{T}\right)$ fit are shown in Fig. 19.

The $7 \mathrm{TeV}$ MC simulation (SHERPA [30]) was analyzed in the same manner yielding $\beta=-0.2 \pm 0.06$, $\alpha=1.5 \pm 0.2 \times 10^{-5}\left(\mathrm{pb} / \mathrm{GeV}^{2}\right) \mathrm{TeV}^{n p T}$ and $n_{p T}=7.1 \pm 0.2$.

Adding the $\beta$ term of Eqs. (12) and (13) improves the $\chi^{2}$ of the $A\left(p_{T}, s\right)$ fits for inclusive jet production quite significantly as noted in Table IX compared with Table II and seen in Fig. 19. However, the data are good enough to draw only rough conclusions about the systematics of the s dependence. The $\beta$ and $n_{p T}$ terms are roughly independent of $\sqrt{ } s$, whereas the $\alpha$ term grows roughly linearly with increasing $s$.

While we have interpreted the deviations from a pure $p_{T}$-power law as real, an uncorrected nonlinearity in the jet energy calibration could also be contributing. The

TABLE IX. The parameters of the quadratic fit for inclusive jets in $\ln \left(p_{T}\right)$ defined by Eq. (13) are tabulated for $\mathrm{p}-\mathrm{p}$ scattering and $\bar{p}$-p of CDF and D0 statistically combined. The fit parameters were determined with $p_{T}$ values in TeV. Correlations between parameters have been neglected.

\begin{tabular}{|c|c|c|c|c|c|}
\hline \multicolumn{6}{|c|}{ QUADRATIC $\operatorname{LN}\left(P_{T}\right)$ FITS } \\
\hline$\sqrt{ } s(\mathrm{TeV})$ & $\beta$ & $\alpha\left(\mathrm{pb} / \mathrm{GeV}^{2}\right) \mathrm{TeV}^{n p T}$ & $n_{p T}$ & $\chi^{2} / d . f$. & d.f. \\
\hline $1.96 \bar{p}-p$ CDF1.96 D0 & $0.03 \pm 0.2$ & $(1.6 \pm 0.8) \times 10^{-6}$ & $6.7 \pm 0.6$ & 0.92 & 38 \\
\hline 2.76 p-p ATLAS & $-0.23 \pm 0.09$ & $\left\{(1.3 \pm 0.6) \times 10^{-6}\right\}$ & $7.5 \pm 0.4$ & 1.17 & 7 \\
\hline 7 p-p ATLAS & $-0.38 \pm 0.05$ & $(1.0 \pm 0.1) \times 10^{-5}$ & $7.8 \pm 0.2$ & 2.50 & 13 \\
\hline 8 p-p CMS & $-0.38 \pm 0.02$ & $(2.1 \pm 0.1) \times 10^{-5}$ & $7.62 \pm 0.05$ & 4.3 & 32 \\
\hline 13 p-p ATLAS & $-0.26 \pm 0.01$ & $(9.2 \pm 0.1) \times 10^{-5}$ & $6.92 \pm 0.02$ & 0.77 & 29 \\
\hline 13 p-p CMS & $-0.32 \pm 0.04$ & $(8.7 \pm 0.2) \times 10^{-5}$ & $7.03 \pm 0.07$ & 0.48 & 26 \\
\hline
\end{tabular}




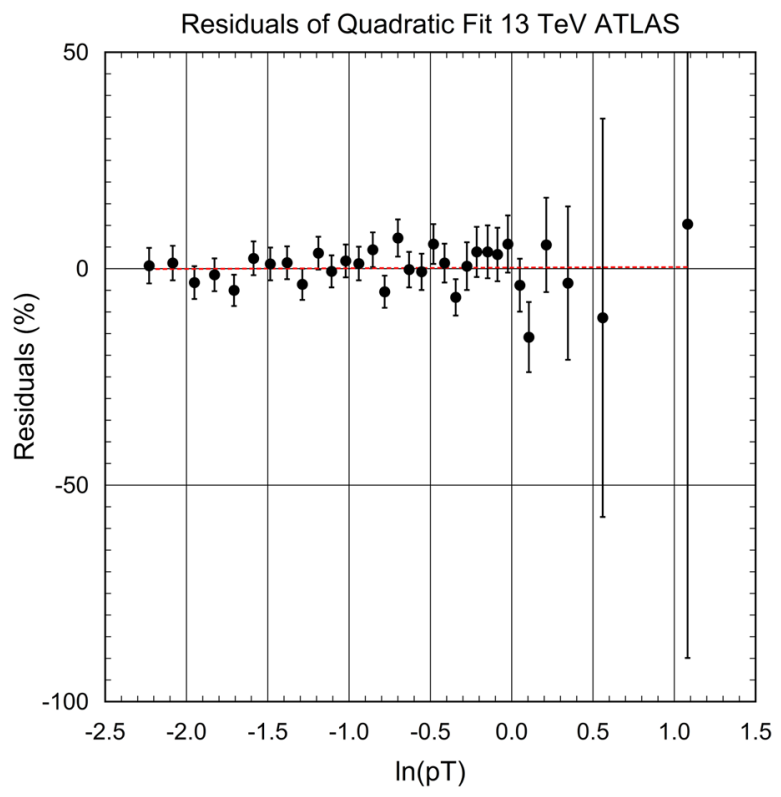

FIG. 19. The residuals of the quadratic $\ln \left(p_{T}\right)$ fit to $13 \mathrm{TeV}$ ATLAS inclusive jet data. Adding the quadratic term in $\ln \left(p_{T}\right)$ improves the $\chi^{2}$ of the fit and reduces the residuals to no further visible dependence on $p_{T}\left(\chi^{2} / d . f .=0.8\right.$ for 29 d.f. $)$. power-law index is independent of an overall energy scale calibration which would only contribute an additive term in the linear fits to $\ln \left(p_{T}\right)$ not affecting the value of $n_{p T}$. However, both the power-law index, $n_{p T}$, as well as a $\beta$ term in Eq. (12) above would be affected by a calorimetry nonlinearity. We note that the form of Eq. (12) is consistent with a log-normal distribution and also a power law modified by a term that is similar to a Sudakov form factor [65] with suitable choice of parameters.

\section{SUMMARY}

This paper is an attempt to characterize inclusive jet production from $\mathrm{p}-\mathrm{p}, \mathrm{p}-\mathrm{Pb}$ and $\bar{p}-p$ collisions at high energy in minimal common terms and to compare these reactions with single-particle inclusive reactions-including charm production and direct photon production at the LHC. Analyzing the invariant cross sections for inclusive jets, single hadrons and prompt photons in $\mathrm{p}-\mathrm{p}$ and $\bar{p}-p$ collisions reveals a simple structure-namely that the invariant cross sections factorize into a product of two power laws, one in $p_{T}$ and the other in $\left(1-x_{R}\right)$. All these inclusive invariant cross sections are of the form given in the equation below,

$$
\begin{aligned}
\frac{d^{2} \sigma}{p_{T} d p_{T} d y}\left(s, p_{T}, y ; \alpha, \Lambda, n_{p T}, m, D, n_{x R 0}\right) & =\frac{\alpha(s)}{\left(\Lambda^{2}+p_{T}^{2}\right)^{n_{p} T}}\left(1-\frac{2 \sqrt{\left(p_{T}^{2} \cosh (y)\left(1+\left(m^{2} / p_{T}^{2}\right) \tanh ^{2}(y)\right)+m^{2}\right)}}{\sqrt{S}}\right)^{\frac{D(s)}{p_{T}}+n_{x_{R} 0}} \\
& =\frac{\alpha(s)}{\left(\Lambda^{2}+p_{T}^{2}\right)^{\frac{n_{p} T}{2}}}\left(1-x_{R}\right)^{\frac{D(s)}{p_{T}}+n_{x_{R} 0}}
\end{aligned}
$$

where the kinematic variables are $\mathrm{s}, p_{T}$ and $\mathrm{y}$ and the parameters are $\alpha, \Lambda, n_{p T}, \mathrm{~m}\left(\right.$ or $\left.m_{J}\right), \mathrm{D}$ and $n_{x R 0}$ described in the text. It is interesting to note that the $\mathrm{s}$ dependence for fixed $x_{R}$ is confined to the parameters $\alpha(s)$ and $\mathrm{D}(\mathrm{s})$, which grow linearly with increasing s. At high $p_{T}\left(p_{T} \gg m\right)$ Eq. (14) simplifies to

$$
\frac{d^{2} \sigma}{p_{T} d p_{T} d y}\left(s, p_{T}, y ; \alpha, \Lambda, n_{p T}, D, n_{x R 0}\right)=\frac{\alpha(s)}{p_{T}^{n_{p T}}}\left(1-\frac{2 p_{T} \cosh (\eta)}{\sqrt{s}}\right)^{\frac{D(s)}{p_{T}}+n_{x_{R} 0}}=\frac{\alpha(s)}{p_{T}^{n_{p T}}}\left(1-x_{R}\right)^{\frac{D(s)}{p_{T}}+n_{x_{R} 0}} .
$$

The $p_{T}$-power laws of Eqs. (14) and (15) are uncovered by using the $x_{R}$ variable to extrapolate the invariant cross sections at various constant $p_{T}$ values as a function of $\left(1-x_{R}\right)$ to the limit $x_{R} \rightarrow 0$ at fixed $\sqrt{ } s$. This procedure determines the underlying $A\left(p_{T}, s\right) \approx \alpha(s) / p_{T}{ }^{n p T}$ function independent of $x_{R}$. All the processes analyzed in this paper have a power-law index confined to $5.3<n_{p T}<7.1$. In broad terms, the $p_{T}$ powers of inclusive cross sections are roughly independent of $\sqrt{ } s$ and process (see the appendix). By averaging all data analyzed (jets, photons, and hadrons) the naive dimensional limit of the invariant cross section $n_{p T}=4$ is disfavored by $3.8 \sigma$. (An even stronger exclusion of $n_{p T}=4$ is obtained by considering the weighted average $\left\langle n_{p T}\right\rangle=6.296 \pm 0.005$.) The data analyzed are consistent with five interacting partons in a $2 \rightarrow 3$ primordial hard scattering that is also a signature of an emergent diquark in the nucleon and that of $2 \rightarrow 2$ scattering with a gluon radiated in one of the final quark lines. A closer examination shows that $A\left(p_{T}, s\right)$ only roughly follows a simple power law in $p_{T}$ for inclusive jets at the LHC. In this case, the $p_{T}$ function is much better fit with a log-normal distribution, or equivalently a power law modified by a term $\sim \exp \left[\beta(s) \ln ^{2}\left(p_{T}\right)\right]$, similar to a Sudakov form factor.

Our procedure involves analyzing the invariant cross sections for a fixed value of $\sqrt{ } s$ in order to determine the $p_{T}$ and the $x_{R}$ dependences. Since the s dependence of the $p_{T}$ and $x_{R}$ dependences has to be estimated by comparing the analysis of different values of $\sqrt{ } s$, it is therefore mandatory to have data sets at several values $(\geq 3)$ of y $(\eta$, or $\theta)$ as well as several values of $p_{T}$ and $\sqrt{ } s$ in order to separate the $p_{T}, x_{R}$ and $\sqrt{ } s$ dependencies. 
The s dependence of our jet fit parameters, shown in Fig. 11 and in Tables I and II, is an indication that jets at low pT and high $\sqrt{ } s$ are strongly quenched. This may be an important factor in planning experiments at a $100 \mathrm{TeV}$ p-p collider.

Without a detailed analysis of the various experimental systematic errors, which is beyond the scope of this work, it is not clear in some cases whether the relatively small differences in parameter values seen are evidence of real differences, such as the different of $p_{T}$ powers of prompt photon production from inclusive jets, or uncorrected systematic effects. Better data and more sophisticated analyses, which for example involve corrections for finite $p_{T}$ and $x_{R}$ bins, would help resolve these issues. In fact, an examination of the fit values, by comparing the parameters for ATLAS and CMS inclusive jets of $n_{p T}$ for inclusive jets in Table I, indicates that $\Delta\left(n_{p T}\right) \sim 0.4$ is within the systematic errors of this analysis.

One aspect of this analysis not explored in detail is the $x_{R}$ dependences. Unlike the $p_{T}$ behavior, the $x_{R}$ side is process, as well as s dependent through the $\mathrm{D}$ term, and is therefore rich phenomenologically. In quark-line counting schemes the exponent of $\left(1-x_{R}\right)$ is dependent on the number of spectator fields and is given by $2 n_{\text {spectator }}-1$ [51]. Examining this feature of the inclusive charm cross section should offer important information about the production mechanism.

The inclusive jet and prompt photon invariant cross sections are well replicated by simulation. In fact, pQCD and various MC programs, such as HERWIG and PHYTHIA $[27,28]$ throughout their historical development, show power laws in $p_{T}$ as well as in the variable $\left(1-x_{R}\right)$. Hence, the elementary behavior revealed in this analysis is already deeply embedded in the simulations and therefore understood. However, the factorized form of the invariant inclusive cross sections, as worked out by this analysis using the $x_{R}$ variable to control phase space, shows a simple structure that may be useful in uncovering nontrivial signatures independent of kinematic effects.

In the original formulation of radial scaling [5], it was posited that all the $\mathrm{s}$ dependence of the inclusive invariant cross sections was in the scaling variable, $x_{R} \approx 2 p_{T} \cosh (\eta) / \sqrt{ } s$, and that the $p_{T}$ and $x_{R}$ dependences of the invariant cross sections completely factorized. This turned out to be not generally true. Data taken at higher collision energies showed that there is an additional $\mathrm{s}$ dependence in the $\alpha(s)$ term, beyond the $x_{R}$ function, that arises from the QCD evolution of the parton, fragmentation and hadronization functions. Moreover, we found in our analysis that the $\left(1-x_{R}\right)$ power index, $n_{x R}$, has a $p_{T}$ dependence that is controlled in our formulation by the $\mathrm{D}$ term. Thus, the rudimentary factorization of the invariant inclusive cross sections into a $p_{T}$ part and an $x_{R}$ part is broken.

The $x_{R}$ variable, unlike $x_{T}$ or $x_{\|}$, has utility in that it quantifies the fraction of the energy of the jet or particle with respect to the kinematic limit in inclusive cross sections that is independent of angle in the COM frame. Controlling this faction breaks the conflation of a purely kinematic effect from a deeper dynamical behavior that seems to have confused several authors. The approximate scaling variable, developed over 40 years ago in the analysis of inclusive particle production in $\mathrm{p}-\mathrm{p}$ collisions, still finds utility in uncovering simple power laws in inclusive jets, photons and charm in both p-p and $\mathrm{p}-\mathrm{Pb}$ collisions at the LHC. Now that the data from the LHC are reaching maturity in broad kinematic ranges, it will be interesting to analyze their broad trends using our formulation.

\section{ACKNOWLEDGEMENTS}

The author thanks his colleagues, especially S. Brodsky, D. Duke, P. Fisher, L. Rosenson and J. Thaler, for interesting discussions and suggestions. The support of the MIT Department of Physics and Laboratory for Nuclear Science is gratefully acknowledged.

\section{APPENDIX: CRITIQUE OF ARLEO et al.}

Arleo et al. [51] have analyzed a number of inclusive measurements, such as inclusive single-particle production in p-p scattering and inclusive jet production at the SPS and FNAL collider. They find that the $p_{T}$ power depends on the process as given in Fig. 20 and is strikingly different from our analysis, which finds all processes examined to be clustered around $n^{\exp } \sim 6.5$. Of particular note is the

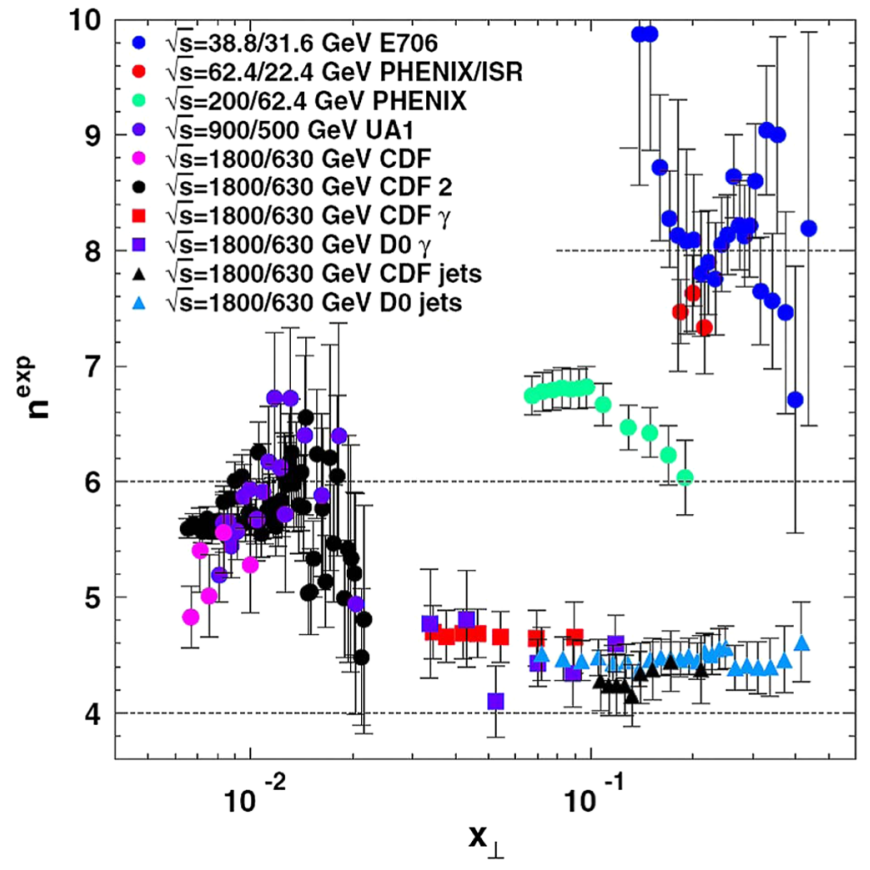

FIG. 20. The effective $p_{T}$ power $n^{\exp }$ from the analysis of Arleo et al. [51] is shown for various processes. This result is strikingly different from this analysis, which finds $n_{p T}$ for all processes examined to be clustered around $n^{\exp } \sim 6.4 \pm 0.5$. Note that the highest exponents determined by Arleo et al. are from comparisons of the lowest $\sqrt{ } s$ data. 
analysis of inclusive jets at CDF and D0 (triangles in the figure below) where the exponent $n^{\exp } \sim 4.5$ is found.

Arleo et al. posit that the invariant cross sections depend on $p_{T}$ and $x_{T}$ defined by $x_{T}=2 p_{T} / \sqrt{ } s$. By computing the ratios of cross sections at different values of $\sqrt{ } s$ they are able to extract the effective $p_{T}$ power denoted by $n^{\exp }$. In their analysis, the invariant cross section is given in Eq. (A1),

$$
\sigma^{\mathrm{inv}} \equiv E \frac{d^{3} \sigma}{d p^{3}}(A B \rightarrow C X)=\frac{F\left(x_{T}, \theta\right)}{p_{T}^{n}}
$$

The analysis rests on the assumption that the function $F\left(x_{T}, \theta_{1}\right) \approx F\left(x_{T}\right)$ and that the s dependence of the cross section is entirely through the $x_{T}=2 p_{T} / \sqrt{ } s$ term so that the invariant cross section can be written as

$$
\sigma^{\mathrm{inv}}(A B \rightarrow C X) \propto \frac{\left(1-x_{T}\right)^{2 n_{\text {spectator }}-1}}{p_{T}^{2 n_{\text {active }}-4}} .
$$

The $n^{\exp }$ value is determined by the ratio of the cross sections and the respective ratio of the COM energies. From Eqs. (A1) and (A2) using $p_{T}=x_{T} \sqrt{ } s / 2$,

$$
n^{\exp }=\frac{-\ln \left(\sigma^{\mathrm{inv}}\left(x_{T}, \sqrt{s_{1}}\right) / \sigma^{\mathrm{inv}}\left(x_{T}, \sqrt{s_{2}}\right)\right)}{\ln \left(\sqrt{s_{1}} / \sqrt{s_{2}}\right)} .
$$

But by the radial scaling hypothesis, the form in Eq. (A2) is generally not true since the function $F\left(x_{T}\right)$ is really a function of $p_{T}, \theta\left(x_{R}\right)$ and importantly of $\sqrt{ } s$ through the $\alpha(s)$ term.

In order to show the flaw in this analysis at least for LHC inclusive jets, we take our parametrization of the LHC inclusive jets at $\sqrt{ } s=13 \mathrm{TeV}$ and $2.76 \mathrm{TeV}$ given in Tables I and II to determine $n^{\exp }$ in the same way. We compute the ratio of 13 to $2.76 \mathrm{TeV}$ ATLAS inclusive jets to examine the cross section ratio as $x_{T} \rightarrow 0$. The result is shown in Fig. 21 where we plot $n^{\exp }$ given by Eq. (A3) as a function of $x_{T}$ for various fixed jet COM angles $\theta$. We find that our evaluation of Eq. (A1) yields an effective $p_{T}$ power of $\sim 4$ in the limit $x_{T} \rightarrow 0$ consistent with the analysis of Arleo et al. [51] for CDF and D0 inclusive jets at 1.8/0.63 TeV. Similar results are obtained when we compare $13 \mathrm{TeV}$ jets to $7 \mathrm{TeV}$ jets.

It is interesting to note that this result for the ATLAS data depends strongly on the s dependence of the cross sections through the $\alpha$ term of Eq. (7). Setting the term $\alpha=1$, but leaving the other parameters $\left(\mathrm{np}_{T}, \mathrm{D}\right.$ and $\left.\mathrm{nx}_{R 0}\right)$ at their fit values one finds $n^{\exp } \approx 6.3$ as $x_{T} \rightarrow 0$. Setting all parameters to the same value ( $\alpha=1, n_{p T}=6.3, D=0$ and $\mathrm{nx}_{R 0}=3.5$ ) we find $n^{\exp } \approx 6.3$. But putting in the measured s dependence of $\alpha$ and leaving the other parameters of the cross section the same $\left(\mathrm{np}_{T}=6.3, D=0\right.$ and $\left.\mathrm{nx}_{R} 0=3.5\right)$ we find $n^{\exp } \approx 4$.4. These results indicate that $n^{\exp } \approx 4$ of Arleo et al. is a result of the s dependence of the cross section, which in our parametrization is mostly through the $\alpha$ term for small $x_{R}$, and not a true measure of the intrinsic $p_{T}$ dependence.
ATLAS Jets $13 \mathrm{TeV} / 2.76 \mathrm{TeV}$

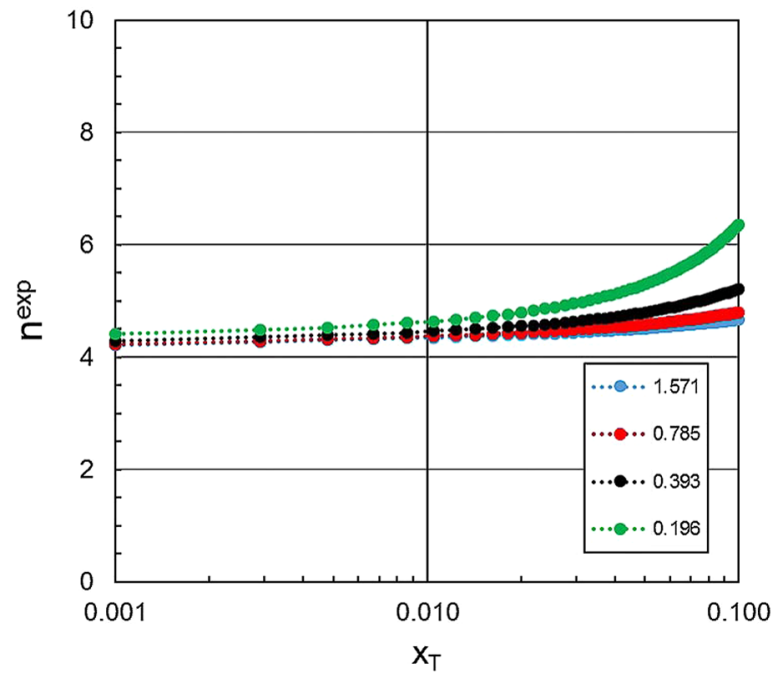

FIG. 21. The ATLAS inclusive jet cross section parametrizations given in Tables I and II for $\sqrt{ } s=2.76$ and $13 \mathrm{TeV}$ are used to evaluate Eq. (A1) above. The various lines are for fixed COM angles starting at $\theta=\pi / 2$ down to 0.196 radians. All lines converge to $n^{\exp } \sim 4.3$ even though the underlying $p_{T}$ dependence is $\sim 1 / p_{T}{ }^{6.3}$.

In order to compute the true $p_{T}$ power exponent, $\mathrm{n}$, of the invariant cross section given in Eq. (A1), we must include not only the $\theta$ dependence, or equivalently the $x_{R}$ dependence of the cross section, but also the $\alpha$-term s dependence. Thus, Eq. (A1) becomes

$\sigma^{\mathrm{inv}} \equiv E \frac{d^{3} \sigma}{d p^{3}}(A B \rightarrow C X)=\frac{\alpha(\sqrt{s})\left(1-x_{R}\right)^{n x_{R}(\sqrt{s}, p T)}}{p_{T}^{n}}$.

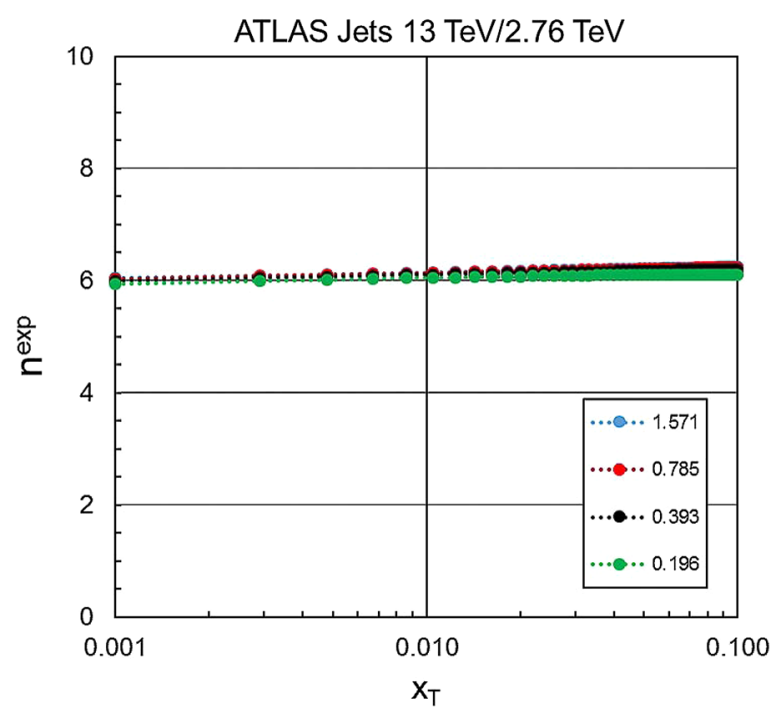

FIG. 22. The effective $p_{T}$ exponent analyzed by the ratio of ATLAS inclusive jets measured at 13 and $2.76 \mathrm{TeV}$ is plotted as a function of $x_{T}$ from Eq. (A5), which includes all the s-dependent terms. We see that the intrinsic $p_{T}$ dependence is correctly calculated. 
In the limit of small $x_{T}\left[x_{T}=x_{R} \sin (\theta)\right]$ Eq. (A4) implies

$$
n^{\exp }=\frac{-\ln \left(\sigma^{\operatorname{inv}}\left(x_{T}, \sqrt{s_{1}}\right) /\left(\sigma^{\text {inv }}\left(x_{T}, \sqrt{s_{2}}\right)\right)+\ln \left(\alpha\left(\sqrt{s_{1}}\right) / \alpha\left(\sqrt{s_{2}}\right)\right)\right.}{\ln \left(\sqrt{s_{1}} / \sqrt{s_{2}}\right)} .
$$

The resultant $n^{\exp }$ is shown in Fig. 22, where it is clear that the $n^{\exp }=6$ is regained with the necessary $\alpha(s)$ term of Eq. (A5) operative (see Fig. 11). Note,

$$
\frac{\ln \left(\alpha\left(\sqrt{s_{1}}\right) / \alpha\left(\sqrt{s_{2}}\right)\right)}{\ln \left(\sqrt{s_{1}} / \sqrt{s_{2}}\right)} \approx 1.9
$$

thereby accounting for the change $n^{\exp } \sim 4$ to $n^{\exp } \sim 6$. Note that the value of Eq. (A6) is a reflection of the $\mathrm{s}$ dependence of $\alpha(\sqrt{ } s) \sim(\sqrt{ } s)^{2}=s$.

Therefore, the method of Arleo et al. [51] determines the effective $p_{T}$ power exponent of ATLAS inclusive jets to be $n_{p T} \sim 4$ (Fig. 21) because the overall $\mathrm{s}$ dependence of the $\alpha$ term of the invariant inclusive cross section has been neglected (Fig. 11 and Table II). It is not unreasonable to conclude that the varying $p_{T}$ power exponents determined in Arleo et al.'s analysis [51] result from the neglect of the $\mathrm{s}$ dependences of the corresponding $\alpha$ terms. Our analysis, which determines the $p_{T}$ dependence at a fixed $\sqrt{ } s$ by extrapolating the $\left(1-x_{R}\right)$ function to $x_{R}=0$, finds $n_{p T} \approx 6.4 \pm 0.5$ for many inclusive measurements over a wide energy range (Tables I, II, III, IV and VIII) and excludes $n_{p T}=$ 4 by $3.8 \sigma$.
[1] R. D. Field and R. P. Feynman, Quark elastic scattering as a source of high-transverse-momentum mesons, Phys. Rev. D 15, 2590 (1977).

[2] R. P. Feynman, R. D. Field, and G. C. Fox, Quantumchromodynamic approach for the large-transversemomentum production of particles and jets, Phys. Rev. D 18, 3320 (1978).

[3] J. F. Owens, E. Reya, and M. Gluck, Detailed quantumchromodynamic predictions for high-pT processes, Phys. Rev. D 18, 1501 (1978).

[4] D. C. Carey et al., Unified Description of Single-Particle Production in pp Collisions, Phys. Rev. Lett. 33, 330 (1974).

[5] F. E. Taylor, D. C. Carey, J. R. Johnson, R. Kammerud, D. J. Ritchie, A. Roberts, J. R. Sauer, R. Shafer, D. Theriot, and J. K. Walker, Analysis of radial scaling in single-particle inclusive reactions, Phys. Rev. D 14, 1217 (1976); Erratum, Phys. Rev. D 15, 3499(E) (1977). Note that at large production angles $(\theta * \sim \pi / 2) \quad x_{F}=p_{\|} /(\sqrt{ } s / 2)$ is not a relevant variable $\left(x_{F} \sim 0\right)$ and the cross sections are usually plotted as a function of $x_{T}=p_{T} /(\sqrt{ } s / 2)$ leading to a somewhat arbitrary choice of relevant scaling variable, $x_{F}$ or $x_{T}$. The radial scaling variable, on the other hand, smoothly goes between these two kinematic regimes since it is approximately $x_{R}=E * / E *_{\operatorname{Max}} \sim\left(x_{F}{ }^{2}+x_{T}{ }^{2}\right)^{1 / 2}$. In the limit of high energy and small particle or jet mass, the radial scaling variable $x_{R} \approx 2 p_{T} \cos (y *) / \sqrt{ } s$.

[6] D. W. Duke and F. E. Taylor, Determination of the sea-quark distributions in the proton by single-particle inclusive reactions, Phys. Rev. D 17, 1788 (1978).

[7] D. C. Carey, J. R. Johnson, R. Kammerud, D. J. Ritchie, A. Roberts, J. R. Sauer, R. Shafer, D. Theriot, J. K. Walker, and F. E. Taylor, Inclusive $\pi^{0}$ production by high-energy protons, Phys. Rev. D 14, 1196 (1976).
[8] K. Eggert et al. A study of high transverse momentum Pi0s at ISR energies, Nucl. Phys. B98, 49 (1975). The $\sqrt{ } s=$ $30.8 \mathrm{GeV}$ data were analyzed by fitting a linear line to $\ln \left(\operatorname{Ed}^{3} \sigma / \mathrm{dp}^{3}\right)$ vs $\ln \left(p_{T}\right)$. The data are sparse in that measurements were made at only two angles, 90 and 53 degrees, so $x_{R}$ lines at constant $p_{T}$ have only two points. This is below the minimum quality of data that were analyzed for inclusive jet/particle production in this paper. Referring to Eqs. (3) and (8), we find $\Lambda=0.80 \pm 0.89 \mathrm{GeV}$ and $n_{p T}=8.1 \pm 3.0$, consistent with lower energy $\pi^{0}$ data.

[9] The simulation of high $p_{T}$ jets is performed by several Monte Carlo programs. See A. Buckley et al., Report No. CERN-PH-TH-2010-298, https://arxiv.org/abs/1101 .2599 .

[10] D. Stump et al., Report No. MSUHEP-030303, 2003, https://arxiv.org/pdf/hep-ph/0303013.pdf.

[11] Amplitude methods: L. J. Dixon, Report No. SLACPUB-15775, 2013, http://www.preposterousuniverse.com/ blog/2013/10/03/guest-post-lance-dixon-on-calculatingamplitudes/.

[12] The ATLAS Collaboration, Report No. ATLAS-CONF2016-092, 2016, https://cds.cern.ch/record/2292095.

[13] The jets were analyzed by the anti- $k_{T}$ algorithm, and used a jet radius $R=0.4$. M. Cacciari, G. Salam, and G. Soyez, The anti- $k_{t}$ jet clustering algorithm, J. High Energy Phys. 04 (2008) 063.

[14] Since the cross section values were not tabulated in the conference note, the data and errors were determined by a scan of Figs. 2 and 3 of [12] using the program ImageJ, https://imagej.nih.gov/ij/.

[15] Z. Nagy, Next-to-leading order calculation of three jet observables in hadron hadron collision, Phys. Rev. D 68, 094002 (2003). 
[16] S. Dulat, T.-J. Hou, J. Gao, M. Guzzi, J. Huston, P. Nadolsky, J. Pumplin, C. Schmidt, D. Stump, and C.-P. Yuan, New parton distribution functions from a global analysis of quantum chromodynamics, Phys. Rev. D 93, 033006 (2016).

[17] D. W. Kolodrubetz et al., Factorization for Jet Radius Logarithms in Jet Mass Spectra at the LHC, arXiv: $1605.08038 \mathrm{v} 1$.

[18] The ATLAS Collaboration, Report No. CERN-PH-EP2013-036, 2013; The ATLAS Collaboration, Measurement of the inclusive jet cross-section in pp collisions at $\sqrt{s}=$ $2.76 \mathrm{TeV}$ and comparison to the inclusive jet cross-section at $\sqrt{s}=7 \mathrm{TeV}$ using the ATLAS detector, Eur. Phys. J. C 73, 2509 (2013).

[19] The ATLAS Collaboration, Measurement of the inclusive jet cross section in proton-proton collisions at $\sqrt{ } s=7 \mathrm{TeV}$ using $4.5 \mathrm{fb}^{-1}$ of data with the ATLAS detector, J. High Energy Phys. 02 (2015) 153.

[20] CDF 1.96 TeV jets: T. Aaltonen et al., Measurement of the inclusive jet cross section at the Fermilab Tevatron $\bar{p} p$ Collider using a cone-based jet algorithm, Phys. Rev. D 78, 052006 (2008); Erratum, Phys. Rev. D 79, 119902 (2009).

[21] D0 $1.96 \mathrm{TeV}$ jets: V. M. Abazov et al., Measurement of the Inclusive Jet Cross Section in $\bar{p} p$ Collisions at $\sqrt{ } s=1.96 \mathrm{TeV}$, Phys. Rev. Lett. 101, 062001 (2008).

[22] CMS 8 TeV jets: The CMS Collaboration, Report No. CMS PAS SMP-14-001, 2015, http://inspirehep.net/record/ 1398651. The jets of this analysis were defined by the anti-kT algorithm with a radius $R=0.7$.

[23] CMS $13 \mathrm{TeV}$ jets: The CMS Collaboration, Measurement of the double-differential inclusive jet cross section in protonproton collisions at $\sqrt{ } s=13 \mathrm{TeV}$, Eur. Phys. J. C 76, 451 (2016). The $R=0.4$ data set was analyzed. The highest $|y|$ bin $(3.2<|y|<4.7)$ was neglected in order to have the $|y|$ range of the CMS data set be the same as the $13 \mathrm{TeV}$ ATLAS data set.

[24] ATLAS 5.02 TeV p-Pb: The ATLAS Collaboration, Centrality, and rapidity dependence of inclusive jet production in $\sqrt{s_{N N}}=5.02 \mathrm{TeV}$ proton-lead collisions with the ATLAS detector, Phys. Lett. B 748, 392 (2015). Data were taken from Tables 2-10, which correspond to integral centrality of $0 \%-90 \%$.

[25] ATLAS 2.76 TeV Pb-Pb: The ATLAS Collaboration, Observation of a Centrality-Dependent Dijet Asymmetry in Lead-Lead Collisions at $\sqrt{ } s_{N N}=2.76 \mathrm{TeV}$ with the ATLAS Detector at the LHC, Phys. Rev. Lett. 105, 252303 (2010).

[26] H. L. Lai, J. Huston, S. Kuhlmann, J. Morfin, F. Olness, J. F. Owens, J. Pumplin, and W. K. Tung (CTEQ Collaboration), Global QCD analysis of parton structure of the nucleon: CTEQ5 parton distributions, Eur. Phys. J. C 12, 375 (2000).

[27] T. Sjostrand, P. Edén, C. Friberg, L. Lönnblad, G. Miu, S. Mrenna, and E. Norrbin, High-energy-physics event generation with Pythia 6.1, Comput. Phys. Commun. 135, 238 (2001); G. Corcella et al., HERWIG 6: an event generator for hadron emission reactions with interfering gluons (including supersymmetric processes), J. High Energy Phys. 01 (2001) 010.

[28] T. Sjostrand, S. Mrenna, and P. Skands, A brief introduction to PYTHIA 8.1, Comput. Phys. Commun. 178, 852 (2008).
[29] R. D. Ball et al., Parton distributions with LHC data, Nucl. Phys. B867, 244 (2013).

[30] SHERPA: S. Höche and M. Schönherr, Uncertainties in next-to-leading order plus parton shower matched simulations of inclusive jet and dijet production, Phys. Rev. D 86, 094042 (2012).

[31] See, for example, David d'Enterria and Juan Rojo, Quantitative constraints on the gluon distribution function in the proton from collider isolated-photon data, Nucl. Phys. B860, 311 (2012).

[32] CMS prompt photons: S. Chatrchyan et al., Measurement of the differential cross section for isolated prompt photon production in $p p$ collisions at $7 \mathrm{TeV}$, Phys. Rev. D 84, 052011 (2011).

[33] ATLAS prompt photons: The ATLAS Collaboration, Measurement of the inclusive isolated prompt photon cross section in $\mathrm{p} p$ collisions at $\sqrt{ } s=8 \mathrm{TeV}$ with the ATLAS detector, J. High Energy Phys. 08 (2016) 005.

[34] ATLAS prompt photons: The ATLAS Collaboration, Measurement of the cross section for inclusive isolated-photon production in $p p$ collisions at $\sqrt{ } s=13 \mathrm{TeV}$ using the ATLAS detector, Phys. Lett. B 770, 473 (2017).

[35] Typically the function Microsoft Excel ® LINEST (https:// support.office.com/en-us/article/LINEST-function-84D7D0 D9-6E50-4101-977A-FA7ABF772B6D) was applied to the logs of the data in order to determine the power-law indices. Having determined trial fit parameters in this way, the $\chi^{2}$ of the fit function vs data was minimized to determine $\Lambda, \alpha$ and $n_{p T}$.

[36] M. M. Mondal and S. Chattopadhyay, in Proceedings of the International Symposium on Nuclear Physics, 2009, pp. 542-543, http://www.sympnp.org/proceedings/54/F4 .pdf.

[37] See, for example, M. Cacciari et al., Theoretical predictions for charm and bottom production at the LHC, arXiv: $1205.6344 \mathrm{v} 1$.

[38] I. C. Arsene et al. (BRAHMS RHIC Collaboration), Kaon and pion production in central $\mathrm{Au}+\mathrm{Au}$ collisions at $\sqrt{ } \mathrm{sNN}=62.4 \mathrm{GeV}$, Phys. Lett. B 687, 36 (2010).

[39] V. Khachatryan et al. The CMS Collaboration, Measurement of $J / \psi$ and $\psi(2 S)$ Prompt Double-Differential Cross Sections in pp Collisions at $\sqrt{ } s=7 \mathrm{TeV}$, Phys. Rev. Lett. 114, 191802 (2015).

[40] The CMS Collaboration, Report No. CMS PAS BPH-15-005, 2016, https://cds.cern.ch/record/2145411/files/BPH-15-005pas.pdf.

[41] C. Albajar et al. (UA1 Collaboration), Direct photon production at the CERN proton-antiproton collider, Phys. Lett. B 209, 385 (1988).

[42] B. Abelev et al. (ALICE Collaboration), Neutral pion production at midrapidity in $\mathrm{pp}$, and $\mathrm{Pb}-\mathrm{Pb}$ collisions at $\sqrt{ } \mathrm{sNN}=2.76 \mathrm{TeV}$, Eur. Phys. J. C 74, 3108 (2014), Table 2.

[43] ALICE Collaboration, Report No. CERN-PH-EP-2015068; Measurement of pion, kaon and proton production in proton-proton collisions at $\sqrt{s}=7 \mathrm{TeV}$, Eur. Phys. J. C 75, 226 (2015).

[44] The LHCb Collaboration, Measurement of prompt charm production cross sections in pp collisions at $\sqrt{ } s=5 \mathrm{TeV}$, arXiv:1610.02230v2. 
[45] The LHCb Collaboration, Measurements of prompt charm production cross-sections in pp collisions at $\sqrt{ } s=13 \mathrm{TeV}$, J. High Energy Phys. 03 (2016) 159.

[46] ATLAS Collaboration, Study of $J / \psi \rightarrow \mu^{+} \mu^{-}$and $\psi(2 S) \rightarrow$ $\mu^{+} \mu^{-}$production with $2015 \mathrm{~Pb}+\mathrm{Pb}$ data at $\sqrt{ } s_{\mathrm{NN}}=$ $5.02 \mathrm{TeV}$ and $\mathrm{p}$ p data at $\sqrt{ } s=5.02 \mathrm{TeV}$ with the ATLAS detector, ATLAS Conf. 109 (2016). Only p-p data were analyzed, https://cds.cern.ch/record/2220771/files/ATLASCONF-2016-109.pdf.

[47] ATLAS Collaboration, Measurement of the differential cross-section of prompt, and nonprompt production of $J / \psi$, and $\psi(2 S)$ in $\mathrm{p} \mathrm{p}$ collisions at $\sqrt{ } s=7$, and $8 \mathrm{TeV}$ with the ATLAS detector, Eur. Phys. J. C 76, 283 (2016).

[48] R. Aaij et al. (LHCb Collaboration), Measurement of forward $J / \psi$ production cross-sections in pp collisions at $\sqrt{s}=13$ TeV, J. High Energ. Phys. 10 (2015) 172.

[49] LHCb Collaboration, Measurement of B meson production cross-sections in proton-proton collisions at $\sqrt{ } s=7 \mathrm{TeV}$, J. High Energy Phys. 08 (2013) 117.

[50] S. J. Brodsky, H. J. Pirner, and J. Raufeisen, Scaling properties of high $\mathrm{pT}$ inclusive hadron production, Phys. Lett. B 637, 58 (2006).

[51] F. Arleo, S. J. Brodsky, D. S. Hwang, and A. M. Sickles, Higher-Twist Dynamics in Large Transverse Momentum Hadron Production, Phys. Rev. Lett. 105, 062002 (2010).

[52] F. Arleo, in Moriond QCD, 2010, http://moriond.in2p3.fr/ QCD/2010/TuesdayMorning/Arleo.pdf.

[53] E. Pohjoisaho, Studying possible higher twist contributions in the inclusive charged hadron cross sections, J. Phys. Conf. Ser. 589, 012016 (2015).

[54] A. Radyushkin, Quark counting rules: Old and new approaches, Int. J. Mod. Phys. A A25, 502 (2010).

[55] E. L. Berger, T. Gottschalk, and D. Sivers, Higher-twist term in inclusive pion production at large transverse momentum, Phys. Rev. D 23, 99 (1981).
[56] M. J. Tannenbaum, Hard and Soft Physics at RHIC with implications for LHC, 4th International Workshop High-pT Physics at LHC 09, 2009, Prague, Czech Republic (2009), Proceedings of Science: https://pos.sissa.it/080/004/pdf.

[57] M. J. Tannenbaum, From the ISR to RHIC - measurements of hard scattering and jets using inclusive single-particle production and 2-particle correlations, http://iopscience.iop .org/article/10.1088/1742-6596/27/1/001.

[58] N. Kochelev, Soft contribution to quark-quark scattering induced by an anomalous chromomagnetic interaction, JETP Lett. 83, 527 (2006).

[59] C.-Y. Wong and G. Wilk, Tsallis fits to $p_{T}$ spectra and multiple hard scattering in pp collisions at the LHC, Phys. Rev. D 87, 114007 (2013).

[60] F. Arleo and D. d'Enterria, Single inclusive pion $p_{T}$ spectra in proton-proton collisions at $\sqrt{ } s=22.4 \mathrm{GeV}$ : Data versus perturbative QCD calculations, Phys. Rev. D 78, 094004 (2008).

[61] A. Selem and F. Wilczek, Hadron systematics and emergent diquarks, edited by G. Grindhammer et al., New Trends in HERA Physics, 2005, pp. 337-356, https://doi.org/10.1142/ 9789812773524_0030.

[62] B. Wojtsekhowski, Nucleon form factors program with SBS at JLAB, arXiv:1401.0859v3.

[63] C. Alexandrou, Ph. de Forcrand, and B. Lucini, Evidence for Diquarks in Lattice QCD, Phys. Rev. Lett. 97, 222002 (2006).

[64] M. Cristoforettia, P. Facciolia, G. Ripkaa, and M. Trainia, Are there diquarks in the nucleon?, Phys. Rev. D 71, 114010 (2005).

[65] V. V. Sudakov, Vertex parts at very high energies in quantum electrodynamics, Sov. Phys. JETP 3, 65 (1956); A. Buckley et al., General-purpose event generators for LHC physics, arXiv:1101.2599v1. 